\title{
Pd(II)-Catalyzed Fujiwara-Moritani Reactions for the Synthesis and Functionalization of Substituted Coumarins
}

\author{
Verónica Ortiz-de-Elguea, Asier Carral-Menoyo, Lorena Simón-Vidal, Mikel Martinez-Nunes, \\ Iratxe Barbolla, Marta G. Lete, Nuria Sotomayor,* and Esther Lete*
}

Cite This: ACS Omega 2021, 6, 29483-29494

Read Online

\section{ACCESS I}

Џlll Metrics \& More

Article Recommendations

Supporting Information

ABSTRACT: Highly substituted coumarins, privileged and versatile scaffolds for bioactive natural products and fluorescence imaging, are obtained via a $\mathrm{Pd}(\mathrm{II})$-catalyzed direct $\mathrm{C}-\mathrm{H}$ alkenylation reaction (Fujiwara-Moritani reaction), which has emerged as a powerful tool for the construction and functionalization of heterocyclic compounds because of its chemical versatility

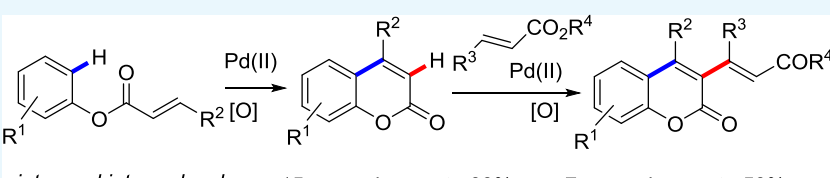

intra and intermolecular 15 examples, up to $83 \% \quad 7$ examples, up to $53 \%$ C-H alkenylations and its environmental advantages. Thus, a selective 6-endo cyclization led to 4-substituted coumarins in moderate yields. Selected examples have been further functionalized in $\mathrm{C} 3$ through a second intermolecular $\mathrm{C}-\mathrm{H}$ alkenylation reaction to give coumarin-acrylate hybrids, whose fluorescence spectra have been measured.

\section{INTRODUCTION}

Coumarins (2H-chromen-2-nes or benzo- $\alpha$-pyrones) are privileged scaffolds of a large variety of natural products ${ }^{1,2}$ (e.g., pestalasins $\mathrm{A}-\mathrm{E}^{3,4}$ and lamellarins ${ }^{5,6}$ ) and/or synthetic molecules, as exemplified by the antioxidant umbelliferone, ${ }^{7}$ choleretic and antispasmodic hymecromone (4-methylumbelliferone), ${ }^{8}$ anti-inflammatory toddaculin, ${ }^{9}$ or anti-HIV agent 4propylcoumarins ${ }^{10}$ (Figure 1). Owing to their outstanding optical properties, these coumarin fluorophores have found applications as fluorescent probes and tracers in biology, laser dyes, solar energy collectors, etc. ${ }^{11-13}$ Additionally, coumarins are widely used as additives in food and cosmetics, ${ }^{14,15}$ as well as agrochemicals, ${ }^{16}$ and pharmaceuticals. ${ }^{17}$ In particular, 4arylcoumarins, a subgroup of flavonoids, have received great attention because they exhibit important biological activities, such as anti-HIV, ${ }^{18}$ antibacterial, ${ }^{19}$ antiprotozoal, ${ }^{20}$ and cytotoxic properties. $^{21}$ For example, 5,7,4'-trimethoxy and 5,7-dimethoxy-4-phenylcoumarins have been tested for their anti-inflammatory activity, specifically as COX inhibitors, which opens the door to future developments as these enzymes are also associated with cancer and neuropsychiatric diseases such as schizophrenia. ${ }^{22}$ The introduction of a fused heterocyclic moiety into the coumarin core usually leads to compounds with promising or even unprecedented properties. ${ }^{23}$ For example, furo[3,2-g]coumarins (e.g., psoralen, xanthoxin, or bergapten) are used in treatment of several skin disorders (psoriasis and vitiligo). ${ }^{24}$ Therefore, the development of synthetic methods to construct and functionalize the coumarin core has attracted much attention over the past decades. Classical approaches for the preparation of coumarin derivatives usually involve the acid- or base-catalyzed condensation reactions of phenol derivatives with esters of acrylic acids (the Pechmann reaction) or with carbonyl compounds (Perkin, Wittig reaction, or Knoevenagel-type reactions). ${ }^{25-27}$ Some milder methods that do not use phenol precursors have also been developed, such as the PIDA/ $\mathrm{I}_{2}$ mediated reactions of substituted phenyl acrylic acids ${ }^{28}$ and visible-light induced synthesis of functionalized coumarins. ${ }^{29}$

Transition-metal-catalyzed, particularly palladium-catalyzed, inter- or intramolecular heteroannulation reactions ${ }^{30,31}$ represent an excellent alternative for the construction of the coumarin framework. ${ }^{32}$ Representative examples include the intramolecular Mizoroki-Heck reaction of $o$-halophenyl 3alkenoates, $^{33}$ the tandem intermolecular Heck/cyclization between aryl halides and acrylates, ${ }^{34-36}$ or the Suzuki crosscoupling/cyclization of 2 -(pinacolboronate)phenol and $\beta$ brominated dehydroaminoacids. ${ }^{37}$ In addition, palladiumcatalyzed intermolecular carbonylative cyclization of $o$ halophenols with alkynes ${ }^{38,39}$ or salicylic aldehydes with benzyl chlorides ${ }^{40}$ has been reported. More recently, Gilmour et al. ${ }^{41}$ has reported a $\operatorname{Pd}(0)$-catalyzed cascade annulation of 2halophenols with $\beta$-borylacrylates as ambiphilic C3-synthons to generate $\mathrm{C} 3$ and $\mathrm{C} 4$-substituted coumarins. However, the transition-metal-catalyzed oxidative coupling reactions ${ }^{42}$ are even more attractive strategies for the synthesis of heterocyclic scaffolds as coumarins, as they avoid the use of preactivated coupling partners (halides, triflates, boronates, etc.). In this

Received: July 2, 2021

Accepted: October 12, 2021

Published: October 25, 2021 


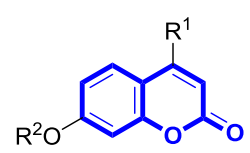

Umbelliferone $\left(R^{1}=R^{2}=H\right)$ Antioxidant

Hymecromone $\left(\mathrm{R}^{1}=\mathrm{CH}_{3}, \mathrm{R}^{2}=\mathrm{H}\right)$ Antispasmodic<smiles>[R]Oc1c2ccoc2c(O[R])c2oc(=O)ccc12</smiles>

Psoralen $\left(R^{1}=R^{2}=H\right)$

Xanthoxin $\left(\mathrm{R}^{1}=\mathrm{H}, \mathrm{R}^{2}=\mathrm{CH}_{3}\right)$ Bergapten $\left(\mathrm{R}^{1}=\mathrm{CH}_{3}, \mathrm{R}^{2}=\mathrm{H}\right)$ Skin diseases<smiles>CCOC(=O)c1cc2cc(/C=C/C(=O)Br)cc(C(C)CC)c2oc1=O</smiles>

Coumarin hybrids Anticancer

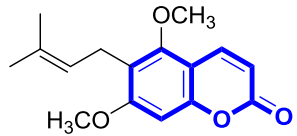

Toddaculin

Anti-inflamatory<smiles>CC=C(C)C(=O)c1c(OC)cc(O)c2c(CCC)cc(=O)oc12</smiles>

4-Alkylcoumarins Anti-HIV activity

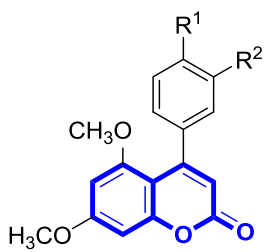

$\mathrm{R}^{1}, \mathrm{R}^{2}=\mathrm{H}, \mathrm{OCH}_{3}, \mathrm{OH}$

4-Arylcoumarins Anti-inflamatory
Figure 1. Selected bioactive compounds with the coumarin core.

context, the oxidative cyclocarbonylation ${ }^{43}$ or carboxylation ${ }^{44}$ of 2-vinylphenols are efficient procedures for the synthesis of 4substituted coumarins. The inter- or intramolecular hydroarylation of alkynes provides a direct and simple route to these heterocycles from phenols and propiolates or aryl propiolates, respectively. ${ }^{45}$ The formation of coumarins by addition of phenols and alkynoates via palladium(II)-catalyzed $\mathrm{C}-\mathrm{H}$ insertion is an excellent example to illustrate the potential of this methodology. ${ }^{46}$ The palladium(II)-catalyzed intermolecular $\mathrm{C}-\mathrm{H}$ alkenylation reaction of phenols and acrylates followed by intramolecular $\mathrm{C}-\mathrm{O}$ formation, first reported by Kitamara et al. $^{47}$ in 2005 and later developed by Maiti et al. ${ }^{48}$ and Shi et $a l^{49}$ has given good results mainly for the synthesis of coumarins with no substituents at the pyrane ring. A related tandem dehydrogenation/oxidative Heck reaction starting from 4-phenylcyclohexanone to generate the intermediate phenol has also been reported. ${ }^{50}$ Therefore, we decided to investigate the intramolecular $\mathrm{C}-\mathrm{H}$ alkenylation variant ${ }^{51,52}$ of aryl alkenoates and cinnamates to prepare 4-alkyl and 4-arylsubstituted coumarins with alkoxy groups at the aromatic ring, a structural feature common to biologically active coumarins (Scheme 1). The strategy involves a 6-endo-trig cyclization, which could be accomplished by controlling the positional selectivity of $\mathrm{C}-\mathrm{H}$ activation, as we have previously demonstrated in the $\mathrm{Pd}(\mathrm{II})$-catalyzed intramolecular 6-endo $\mathrm{C}-\mathrm{H}$ alkenylation of $\mathrm{N}$-arylacrylamides ${ }^{53}$ and $\mathrm{N}$-substituted $\mathrm{N}$ allylanilines $^{54}$ for the construction of quinolone and dihydroquinoline cores, respectively. The effect of the substitution patterns of the benzene ring and the alkene on the reactivity and regioselectivity would be investigated. In addition, as conjugation of coumarin nucleus with an unsaturated moiety at the $\mathrm{C}-3$ position can play a pivotal role in the anticancer activity of these heterocycles, ${ }^{55}$ we
Scheme 1. Synthesis of Coumarins via $\mathrm{C}-\mathrm{H}$ Alkenylation

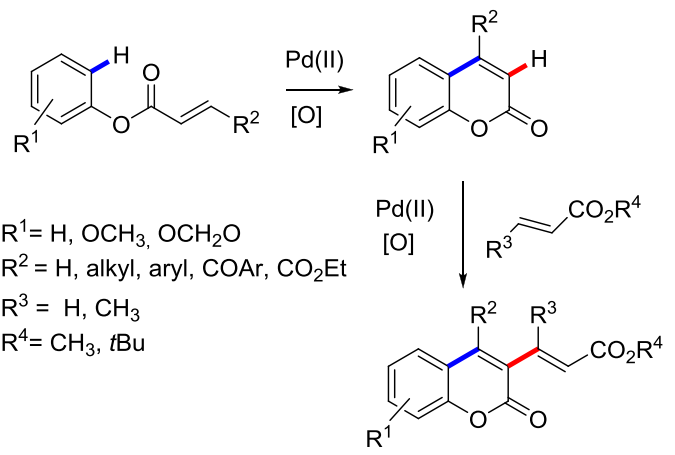

decided to carry out an intermolecular alkenylation reaction on the coumarin ring with $\alpha, \beta$-unsaturated esters to generate new coumarin-acrylate hybrids.

\section{RESULTS AND DISCUSSION}

We started the study of the reaction conditions (palladium catalyst and oxidant) using cinnamate $\mathbf{1 a}$ as the model substrate (Table 1). We selected the reaction conditions that

Table 1. Optimization of 6-Endo Cyclization of 1a<smiles>COc1cc(OC)cc(OC(=O)/C=C/c2ccccc2)c1</smiles>

1a<smiles>COc1cc(OC)c2c(-c3ccccc3)cc(=O)oc2c1</smiles>

$2 \mathbf{a}$
L1:<smiles>CC(=O)c1cccnc1</smiles>

\begin{tabular}{clllll} 
entry & \multicolumn{1}{c}{$[\mathrm{Pd}]$} & \multicolumn{1}{c}{$[\mathrm{O}]$} & $\mathrm{L}$ & $t(\mathrm{~h})$ & 2a (\%) \\
1 & $\mathrm{Pd}(\mathrm{OAc})_{2}{ }^{a}$ & $\mathrm{Cu}(\mathrm{OAc})_{2}{ }^{b}$ & - & 30 & $-{ }^{c}$ \\
2 & $\mathrm{Pd}(\mathrm{OAc})_{2}{ }^{a}$ & $p-\mathrm{BQ}^{b}$ & - & 30 & $-{ }^{c}$ \\
3 & $\mathrm{Pd}(\mathrm{OAc})_{2}{ }^{a}$ & $\mathrm{PhCO}_{3} t \mathrm{Bu}^{d}$ & - & 24 & 9 \\
4 & $\mathrm{Pd}\left(\mathrm{dba}_{2}{ }^{a}\right.$ & $\mathrm{PhCO}_{3} t \mathrm{Bu}^{d}$ & - & 24 & 5 \\
5 & $\mathrm{PdCl}_{2}\left(\mathrm{CH}_{3} \mathrm{CN}\right)_{2}{ }^{a}$ & $\mathrm{PhCO}_{3} t \mathrm{Bu}^{d}$ & - & 8 & $10^{e}$ \\
6 & $\mathrm{PdCl}_{2}\left(\mathrm{CH}_{3} \mathrm{CN}\right)_{2}{ }^{a}$ & $\mathrm{PhCO}_{3} t \mathrm{Bu}^{d}$ & - & 24 & $-{ }^{\prime}$ \\
7 & $\mathrm{PdCl}_{2}\left(\mathrm{CH}_{3} \mathrm{CN}\right)_{2}{ }^{a}$ & $\mathrm{PhCO}_{3} t \mathrm{Bu}^{d}$ & - & $4^{g}$ & $f$ \\
8 & $\mathrm{PdCl}_{2}\left(\mathrm{CH}_{3} \mathrm{CN}\right)_{2}{ }^{a}$ & $\mathrm{PhCO}_{3} t \mathrm{Bu}^{d}$ & $\mathrm{L1}^{a}$ & 24 & 23 \\
9 & $\mathrm{PdCl}_{2}\left(\mathrm{CH}_{3} \mathrm{CN}\right)_{2}{ }^{h}$ & $\mathrm{PhCO}_{3} t \mathrm{Bu}^{d}$ & - & 24 & 25 \\
10 & $\mathrm{PdCl}_{2}\left(\mathrm{CH}_{3} \mathrm{CN}\right)_{2}{ }^{a}$ & $\mathrm{PhCO}_{3} t \mathrm{Bu}^{d}$ & - & $24^{i}$ & 25 \\
11 & $\mathrm{PdCl}_{2}\left(\mathrm{CH}_{3} \mathrm{CN}\right)_{2}{ }^{a}$ & $\mathrm{~F}^{+d, j}$ & - & 24 & 74
\end{tabular}

${ }^{a} 5 \mathrm{~mol} \% .{ }^{b} 1$ equiv. ${ }^{c}$ Starting material was recovered. ${ }^{d} \mathrm{Cu}(\mathrm{OAc})_{2}(5$ mol \%) was used as a co-oxidant. ${ }^{e}$ Conversion: $31 \% .{ }^{f}$ Decomposition. ${ }^{g_{\text {The }}}$ reaction was carried out under reflux. ${ }^{h} 10 \mathrm{~mol} \%$. ${ }^{i} \%$ wt. PT aqueous solution was used as solvent instead of HOAc. ${ }^{j} N$-Fluoro2,4,6-trimethylpyridinium triflate (1.2 equiv) was used as the oxidant

had been previously employed for the 6-endo cyclization of $\mathrm{N}$ aryl acrylamides to quinolones ${ }^{53}$ as a starting point, carrying out the reactions at room temperature in acetic acid and in the presence of $p-\mathrm{TsOH}$ ( 1 equiv) as an additive. The presence of this acid has been shown to be a determinant to increase the reactivity in this type of reaction. ${ }^{53,54,56,57}$ However, when palladium acetate was used as the catalyst using copper acetate or $p$-benzoquinone as oxidants, no conversion at all was observed after $30 \mathrm{~h}$ at $\mathrm{rt}$, recovering unreacted 1a (Table 1, entries 1 and 2 ). The use of t-butyl perbenzoate in combination with copper acetate as an oxidant was required 
to obtain some reactivity, although low yields of the corresponding coumarin $2 \mathrm{a}$ were isolated, observing also the formation of decomposition products (Table 1, entries 3 and 4). A change in the palladium catalyst to bis(acetonitrile)dichloropalladium(II) resulted in a low conversion of the substrate (69\% of 1a was recovered) after $8 \mathrm{~h}$, but with a low isolated yield of $\mathbf{2 a}$ (Table 1, entry 5). A longer reaction time or higher reaction temperature was detrimental due to decomposition of the substrate (Table 1, entries 6 and 7). The use of a pyridine ligand (L1), a higher catalyst loading (10 mol \%), or the use of an aqueous medium for the reaction resulted in increased but still low isolated yields (Table 1, entries 8-10). In no case, open-ring or decarboxylated products could be observed in isolable quantities. It was necessary to use a stronger oxidant such as $N$-fluoro-2,4,6trimethylpyridinium triflate $\left(\mathrm{F}^{+}\right)$to obtain a complete conversion after $24 \mathrm{~h}$, affording coumarin $2 \mathrm{a}$ in good isolated yield (Table 1, entry 11). This oxidant has been successfully employed in related intermolecular $\mathrm{C}-\mathrm{H}$ alkenylation reactions in which the use of weaker oxidants gave low reactivity. ${ }^{58-60}$ It is noteworthy that the reaction was completely regioselective toward the formation of the 6-endo cyclization product (coumarin $\mathbf{2 a}$ ), not observing the formation of the corresponding 5-exo cyclization product, benzofuran-2(3H)-one. Finally, a control experiment was carried out and in the absence of palladium, the reaction did not take place and unreacted 1a was recovered after $24 \mathrm{~h}$ at $\mathrm{rt}$.

These reaction conditions were applied to a series of cinnamates and enoates $\mathbf{1} \mathbf{b}-\mathbf{1} \mathbf{k}$. The results are summarized in Table 2.

As shown, the results obtained were highly dependent on the substrate employed. Different substitution patterns on the aromatic ring at $\mathrm{C} 4$ are tolerated, and coumarins $\mathbf{2 b}-\mathbf{d}$ were obtained in good isolated yields (Table 2). Nevertheless, the obtention of $2 \mathrm{e}$ required the use of a higher catalyst loading (10 mol \%). A heteroaromatic ring, such as furan, could also be introduced at $\mathrm{C} 4$, although in a lower yield (2f, $21 \%)$. When an electron-deficient aryl group was introduced, $\mathbf{2 g}$ was obtained in a much lower yield (28\%). Interestingly, when the reaction temperature was increased to $70{ }^{\circ} \mathrm{C}$, the regioselectivity of the reaction was lost, isolating both the 6endo and 5-exo cyclization products ( $2 \mathrm{~g}$ and $3 \mathrm{~g}$, respectively) in low yields, together with decomposition products. Under these reaction conditions, unsubstituted or 4-alkyl substituted coumarins $\mathbf{2} \mathbf{h}-\mathbf{k}$ were obtained only in moderate to low yields (Table 2), though no starting material could be recovered. An increase of the reaction temperature to $70{ }^{\circ} \mathrm{C}$ did not improve the results. It was also shown that the reaction conditions are not compatible with the presence of acyl groups on the alkene $\left(11 \mathrm{R}^{1}=\mathrm{CO}_{2} \mathrm{Et} ; 1 \mathrm{~m} \mathrm{R} \mathrm{R}^{1}=\mathrm{COAr}\right)$ or with the substitution on the $\alpha$-position of the alkene ( 1 n and $10 \mathrm{R}=\mathrm{Ph}$ and $\mathrm{CH}_{3}$, respectively). In these cases, complex mixtures of products were obtained, not isolating coumarins 21-2o. These results show that it is possible to access the coumarin framework via 6-endo cyclization, but this procedure is not as efficient as it was shown for the synthesis of quinolones from the corresponding amides. ${ }^{53}$ This difference in reactivity could be attributed to the instability of the esters in acidic media, leading to decomposition when long reaction times are required. Consequently, the reaction conditions were reevaluated to use an alternative solvent to acetic acid, using in this case pent-5-enoate $\mathbf{1} \mathbf{j}$ as the model substrate (Table 3).
Table 2. Synthesis of 4-Substituted Coumarins $2 \mathrm{a}-\mathrm{g}$
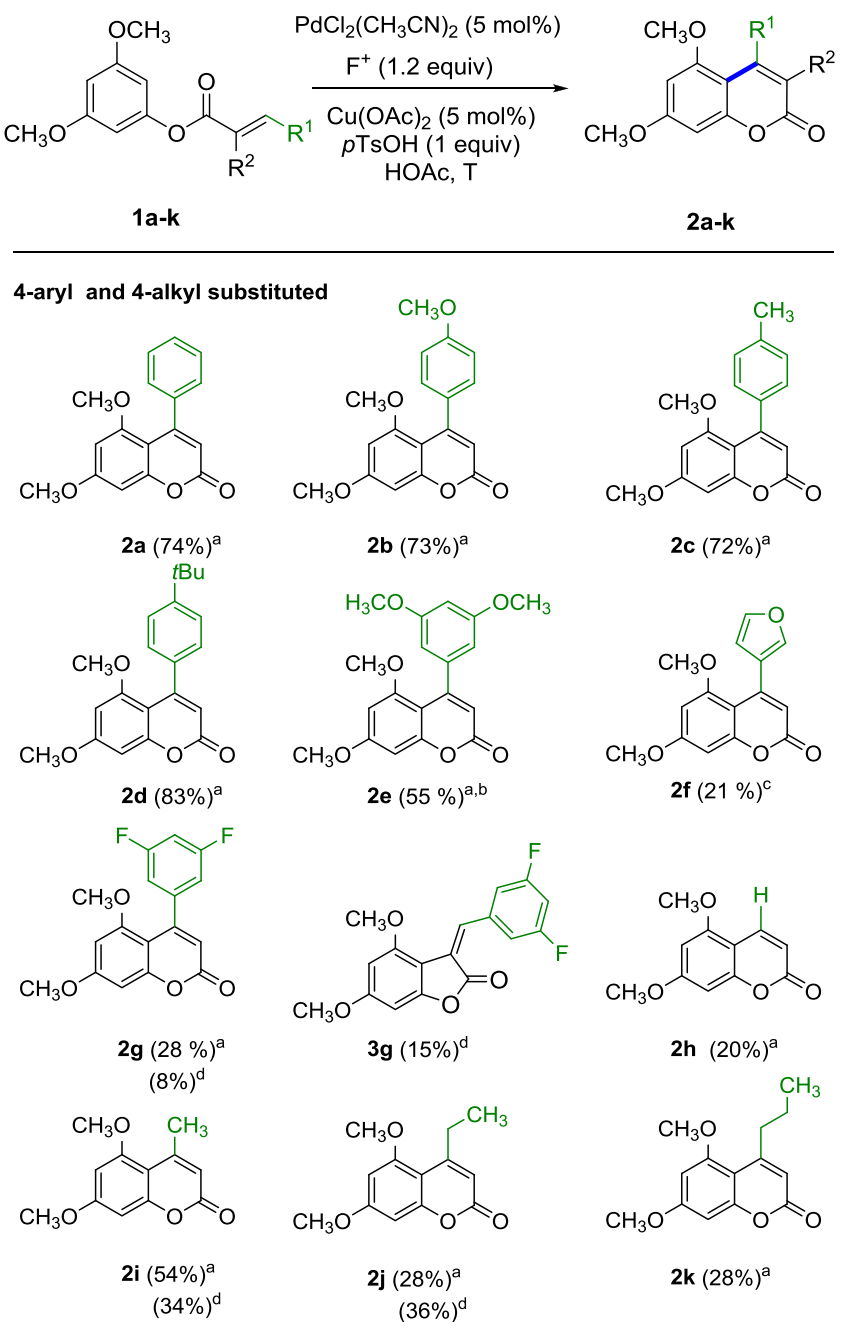

4-acyl and 3,4-disubstituted: non compatible

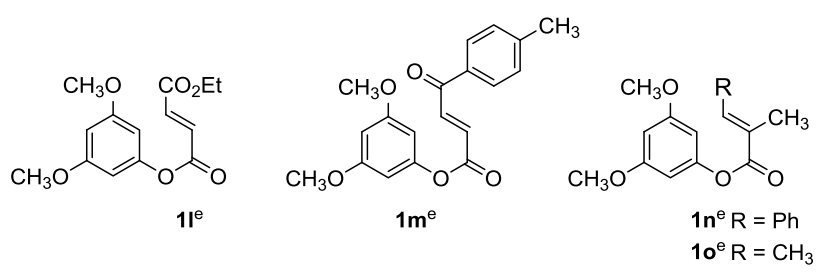

${ }^{a}$ Yield (\%) of the pure isolated compound (rt, 6-24 h). ${ }^{b} 10 \mathrm{~mol} \%$ of the catalyst was used; $79 \%$ conversion. ${ }^{c} 75 \%$ conversion. ${ }^{d}$ Yield (\%) of the pure isolated compound $\left(70^{\circ} \mathrm{C}, 24 \mathrm{~h}\right) .{ }^{e}$ Decomposition.

The change of acetic acid for a non-protic solvent, such as dichloromethane, led to a low conversion at room temperature (Table 3, entry 1). The use of an apolar solvent, such as mesitylene, in combination with a smaller proportion of acetic acid $^{61}$ led to an increase of the isolated yield of $2 \mathbf{j}$, although in a longer reaction time (Table 3 , entry 2 ). The reactivity could be recovered at $40{ }^{\circ} \mathrm{C}$, with slight erosion of the yield (Table 3, entry 3 ). When the reaction was carried out in mesitylene, the presence of $p-\mathrm{TsOH}$ was essential, as no reactivity was observed even after $96 \mathrm{~h}$ at $\mathrm{rt}$ in its absence (Table 3, entry 4). Finally, a moderate yield of $2 \mathrm{j}$ could be obtained at $70{ }^{\circ} \mathrm{C}$ (Table 3, entry 5). The use of these reaction conditions (mesitylene, $70{ }^{\circ} \mathrm{C}$ ) resulted in a significant increase in the 
Table 3. Cyclization Reaction Conditions for $\mathbf{1 j}$

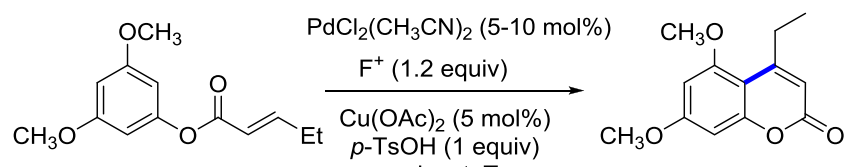

$1 \mathrm{j}$

solvent, T

\begin{tabular}{clccc|}
\hline entry & \multicolumn{1}{c}{ solvent } & $T\left({ }^{\circ} \mathrm{C}\right)$ & $t(\mathrm{~h})$ & $\mathbf{2 j}(\%)$ \\
\hline $1^{a}$ & $\mathrm{CH}_{2} \mathrm{Cl}_{2}$ & $\mathrm{rt}$ & 24 & $8^{b}$ \\
$2^{c}$ & mesitylene/HOAc $4: 1$ & $\mathrm{rt}$ & 96 & 37 \\
$3^{c}$ & mesitylene/HOAc $4: 1$ & 40 & 24 & 33 \\
$4^{c}$ & mesitylene & $\mathrm{rt}$ & 96 & $-^{d}$ \\
$5^{c}$ & mesitylene & 70 & 24 & 49 \\
\hline
\end{tabular}

${ }^{a} 5 \mathrm{~mol} \%$ of catalyst. ${ }^{b}$ Conversion: $17 \% .{ }^{c} 10 \mathrm{~mol} \%$ of catalyst. ${ }^{d}$ No $p$ $\mathrm{TsOH}$ was added; no reaction.

yield of coumarin $\mathbf{2 h}$ (69\% vs 20\%) and 4-alkyl substituted coumarins $\mathbf{2} \mathbf{i}-\mathbf{2 k}$ (Table 4 ). The yield of 4 -aryl substituted

Table 4. Synthesis of 4-Substituted Coumarins in Mesitylene

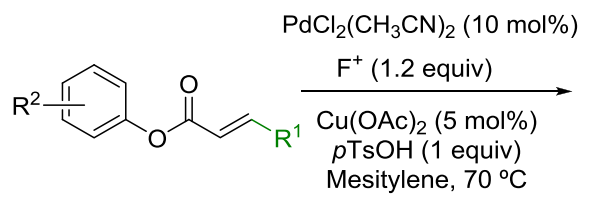

1<smiles>COc1cc(OC)c2ccc(=O)oc2c1</smiles>

2h $(69 \%)^{a, b}$<smiles>CCCc1cc(=O)oc2cc(OC)cc(O)c12</smiles>

2k $(50 \%)^{\text {a }}$<smiles>COc1cc2oc(=O)cc(C)c2cc1OC</smiles>

2q $(34 \%)^{a}$<smiles>COc1cc(OC)c2c(C)cc(=O)oc2c1</smiles>

$2 \mathbf{i}(60 \%)^{a}$<smiles>COc1cc(OC)c2c(-c3cc(F)cc(F)c3)cc(=O)oc2c1</smiles>

2g $(30 \%)^{a}$

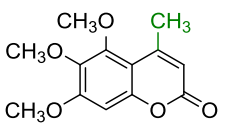

$2 \mathbf{r}(18 \%)^{\mathrm{a}}$

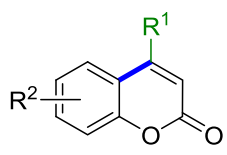

2

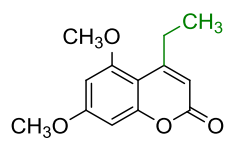

2j $(49 \%)^{a}$
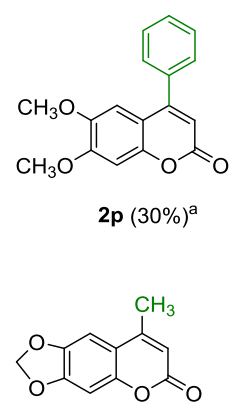

2s $(20 \%)^{\mathrm{a}}$
${ }^{a}$ Yield (\%) of the pure isolated compound. ${ }^{b} 5$ mol\% of catalyst.

coumarin $2 \mathrm{~g}$ could not be improved under these conditions, although in this case, the formation of the 5-exo product $3 \mathrm{~g}$ was not observed by NMR. Finally, the extension to different substitution patterns in the aromatic ring was also studied (Table 4). It was clear that an electron-rich aromatic ring is a determinant to obtain reactivity under these conditions.

Two mechanistic pathways could be proposed for this type of oxidative alkenylation reaction. On one hand, arene metalation with an electrophilic $\mathrm{Pd}(\mathrm{II})$ species would give an aryl-Pd(II) species I, followed by syn-migratory insertion onto the alkene to give II, and syn elimination, after isomerization to
III, to afford coumarin $2 .{ }^{53}$ Alternatively, the alkene could be first activated by the palladium catalyst (IV), followed by antiaddition of the aromatic ring to give also III, and syn elimination would lead to 2 (Scheme 2). In both cases, an electron-rich aromatic ring would be more reactive. As shown on Table 4, 6,7-dialkoxycoumarins $\mathbf{2 p}, \mathbf{2 q}$, and 2 s could be obtained regioselectively, although in lower yields, as the 5,6,7trimethoxy-substituted coumarin $2 \mathbf{r}$, but no unreacted substrate was recovered. Although other aromatic substitution patterns were tested, no reactivity was observed with 3,5dimethylphenyl, 3-methoxyphenyl, and 3-methoxy-5-methyl cinnamates, while complex mixtures of products were formed when using the corresponding (E)-but-2-enoates (see the Supporting Information for the substrates tested, 1t-1y).

The selective functionalization of the coumarin skeleton is also an important issue as, in addition to the biological properties, the extension of the conjugation could affect, for instance, their photophysical properties. ${ }^{62,63}$ Thus, several procedures have been developed for the C3 alkenylation of coumarins. For instance, palladium-catalyzed decarboxylative couplings have been described for the functionalization of coumarins with cinnamic acids ${ }^{64}$ and for the reaction of coumarin-3-carboxylic acid with acrylates. ${ }^{65}$ In addition, the selective $\mathrm{C} 3$ alkenylation of non-substituted coumarins has also been efficiently accomplished through the oxidative Heck reaction. $^{66}$ Thus, we decided to study the derivatization of selected 4-substituted coumarins through a second intermolecular $\mathrm{C}-\mathrm{H}$ alkenylation reaction.

First, we tested the conditions used for the 6-endocyclization, but no intermolecular alkenylation reaction with $t$-butyl acrylate was observed. After some experimentation, we found that the best results were obtained using the reaction conditions previously developed for the alkenylation of quinolones. ${ }^{53}$ However, in most cases, conversions were low and $30-40 \%$ of the starting material was recovered (Table 5). When longer reaction times or higher temperatures were used, complex mixtures of products were isolated. We tried to optimize the reaction conditions by changing the oxidant or the palladium source, but the results could not be improved (see the Supporting Information). As shown in Table 5, C4 aryl- and methyl-substituted coumarins $\mathbf{2 a}, \mathbf{d}, \mathbf{e}, \mathbf{i}$ could be alkenylated with methyl or $t$-butyl acrylate using palladium acetate in the presence of silver acetate and pivalic acid obtaining alkenylated coumarins $\mathbf{4 a}-\mathbf{f}$ in moderate yields (Table 5, entries 1-6). Substitution on the $\beta$-position of the alkene is also possible, obtaining $\mathbf{4 g}$ in moderate yield (Table 5, entry 7).

As 3-vinyl and 3-styryl coumarins ${ }^{62,63}$ can be interesting fluorophores, the absorption and emission properties of selected coumarins $\mathbf{2 a , d , e}$ and $\mathbf{4 a}-\mathbf{d}$ were also studied (see Table 6, Figure 2, and the Supporting Information). All coumarins showed UV-vis absorption, only one absorption band around $\lambda_{\text {max. }}=375 \mathrm{~nm}$ for 4-aryl coumarins $\mathbf{2 a , d , e ~ a n d ~}$ around $\lambda_{\text {abs. }}=300 \mathrm{~nm}$ for their C3 alkenylated derivatives $4 \mathbf{a}-$ d. This shift to a lower wavelength upon introduction of the acrylate moiety reflects the loss of coplanarity of the aromatic ring and the coumarin nucleus, thus decreasing delocalization. C3 alkenylation in the coumarin core leads to considerable changes in the fluorescence properties of coumarins $4 a-d$, whose maximum emission were more blue-shifted than those of coumarins $2\left(\lambda_{\mathrm{em} .}=452-480 \mathrm{~nm}\right.$ vs $\left.\lambda_{\mathrm{em} .}=435-446 \mathrm{~nm}\right)$ and presented higher Stoke shifts (up to 180) (Table 6). 
Scheme 2. Mechanistic Pproposals for the 6-Endo Cyclization: Arene Metalation vs Alkene Activation

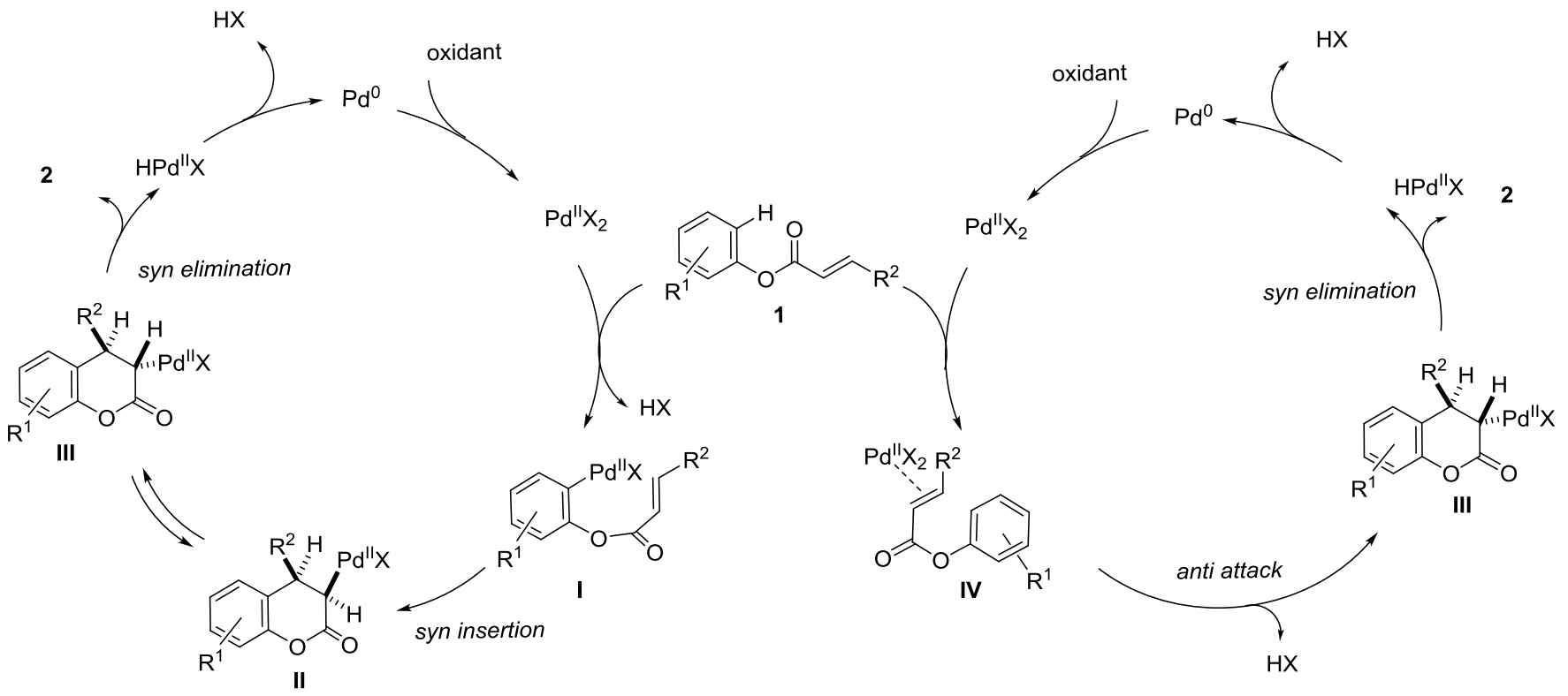

Table 5. Intermolecular $\mathrm{C}-\mathrm{H}$ Alkenylation of Coumarins 2

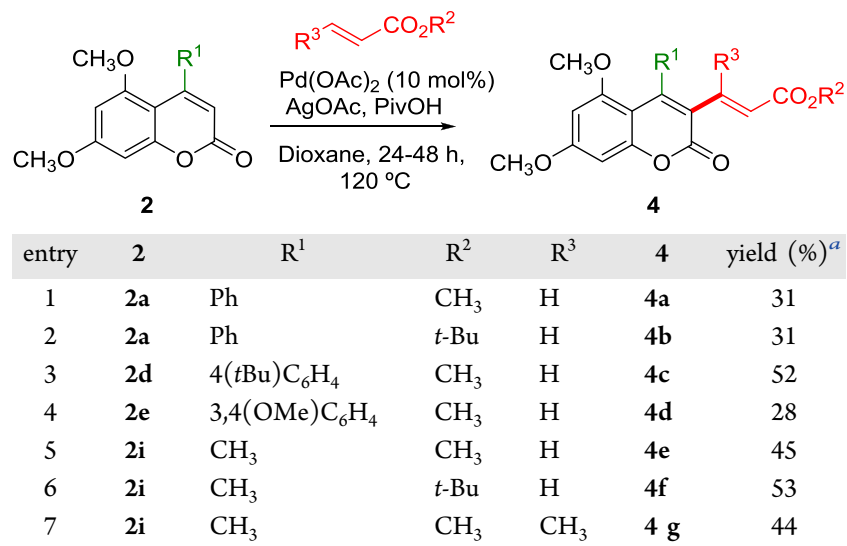

${ }^{a}$ Yield (\%) of the pure isolated compound; in all cases, $30-40 \%$ of the unreacted starting material was also recovered.

Table 6. Absorption and Emission Maxima of Selected Coumarins 2 and 4

$\begin{array}{ccccc}\text { entry } & \text { compd } & \lambda_{\mathrm{abs}}{ }^{a}(\mathrm{~nm}) & \lambda_{\mathrm{em}}{ }^{b}(\mathrm{~nm}) & \Delta \text { Stoke }^{c}(\mathrm{~nm}) \\ 1 & \text { 2a } & 375 & 435 & 60 \\ 2 & \text { 2d } & 377 & 441 & 64 \\ 3 & \text { 2e } & 376 & 446 & 70 \\ 4 & \text { 4a } & 301 & 453 & 152 \\ 5 & \text { 4b } & 302 & 452 & 150 \\ 6 & \text { 4c } & 300 & 480 & 180 \\ 7 & \text { 4d } & 300 & 455 & 155\end{array}$

${ }^{a}$ The maximum absorption wavelength in acetonitrile $(2.3 \mathrm{mM})$. ${ }^{b}$ Excited at the maximum absorption wavelength in acetonitrile $(2.3$ $\mathrm{mM}) .{ }^{c}$ Stoke shift $=\lambda_{\mathrm{em}}-\lambda_{\mathrm{abs}}$.

Fluorescent organic dyes with large Stoke shift are essential for biological applications. ${ }^{67-69}$

In conclusion, it has been shown that 4-aryl-substituted coumarins can be obtained in moderate to good yields via palladium(II)-catalyzed $\mathrm{C}-\mathrm{H}$ alkenylation reactions. 4-Arylsubstituted derivatives can be obtained in acetic acid at room temperature, while mesitylene at $70{ }^{\circ} \mathrm{C}$ is required to obtain moderate yields of 4-alkyl-substituted coumarins, avoiding decomposition in an acidic medium. However, the procedure is limited to an electron-rich aromatic ring for the cyclization to proceed efficiently. The coumarin skeleton can be further functionalized via C3 intermolecular alkenylation. Additionally, the fluorescence spectra of selected coumarins have been measured to provide perspective for potential applications.

\section{EXPERIMENTAL SECTION}

General Experimental Methods. Melting points were measured in unsealed capillary tubes. The NMR spectra were obtained at $300 \mathrm{MHz}$ for ${ }^{1} \mathrm{H}$ and $75.5 \mathrm{MHz}$ for ${ }^{13} \mathrm{C}$ or at 500 $\mathrm{MHz}$ for ${ }^{1} \mathrm{H}$ and $125.7 \mathrm{MHz}$ for ${ }^{13} \mathrm{C}$ using $\mathrm{CDCl}_{3}$ as solvent and internal standard at $20-25{ }^{\circ} \mathrm{C}$. Distortionless enhancement by polarization transfer (DEPT) experiments and $2 \mathrm{D}$ correlation experiments (COSY, heteronuclear single-quantum coherence (HSQCed), or heteronuclear multiple bond correlation $(\mathrm{HMBC}))$ were used when necessary for the assignments of individual ${ }^{13} \mathrm{C}$ and ${ }^{1} \mathrm{H}$ resonances. Electron impact (EI, $70 \mathrm{eV})$, chemical ionization $(\mathrm{CI}, 230 \mathrm{eV})$, or electrospray ionization $\left(\mathrm{ESI}^{+}\right)$sources were used for obtaining the mass spectra. A TOF detector was employed for the obtention of exact mass. The IR spectra were obtained using an ATR instrument. The fluorescence spectra were measured using a Jasco FP-6500 spectrofluorimeter using acetonitrile as solvent at $2.3 \mathrm{mM}$. The emission spectra were acquired by irradiating the sample at its maximum absorbance. Both excitation and emission spectra were collected with a $3 \mathrm{~nm}$ slit bandwidth and were recorded at $25{ }^{\circ} \mathrm{C}$. TLC was carried out with $0.2 \mathrm{~mm}$-thick silica gel plates. Visualization was accomplished by UV light. Flash column chromatography was carried out on silica gel (230-400 mesh). All solvents used in reactions were purified according to standard procedures. Palladium catalysts were purchased from Aldrich and were used without further purification: $\mathrm{Pd}(\mathrm{OAc})_{2}, 98 \%$ purity, and $\mathrm{PdCl}_{2}\left(\mathrm{CH}_{3} \mathrm{CN}\right)_{2}$, 99\% purity.

Intramolecular Alkenylation of Enoates 1 and Synthesis of Coumarins 2: General Procedure. 1-Fluoro2,4,6-trimethylpyridinium trifluoromethanesulfonate (1.2 $\mathrm{mmol}), \mathrm{Cu}(\mathrm{OAc})_{2}(0.05 \mathrm{mmol}), p-\mathrm{TsOH}(1 \mathrm{mmol})$, and 


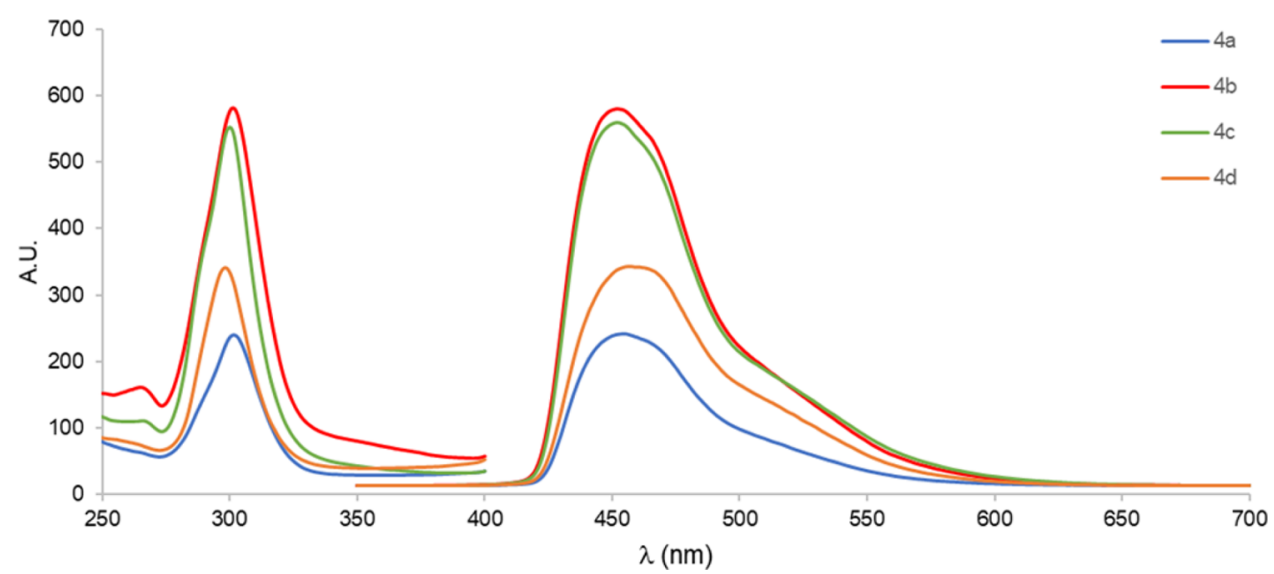

Figure 2. Excitation and emission spectra of coumarins $4 \mathrm{a}-\mathbf{d}$ at $2.3 \mathrm{mM}$ in acetonitrile.

$\mathrm{PdCl}_{2}\left(\mathrm{CH}_{3} \mathrm{CN}\right)_{2}(0.05$ or $0.1 \mathrm{mmol})$ were added sequentially over a solution of the corresponding enoate $1(1 \mathrm{mmol})$ in HOAc or mesitylene $(4 \mathrm{~mL})$. The mixture was stirred at room temperature or at $70{ }^{\circ} \mathrm{C}$ for $4-24 \mathrm{~h}$. The solvent was removed under vacuum and the residue was dissolved in EtOAc $(5 \mathrm{~mL})$. The solution was washed with a $2 \mathrm{M}$ aqueous solution of $\mathrm{Na}_{2} \mathrm{CO}_{3}(2 \times 15 \mathrm{~mL})$ and brine $(2 \times 15 \mathrm{~mL})$. The aqueous phase was re-extracted with EtOAc $(10 \mathrm{~mL})$ and the combined organic extracts were dried $\left(\mathrm{Na}_{2} \mathrm{SO}_{4}\right)$ and concentrated in vacuo. Flash column chromatography (silica gel, hexane/ EtOAc) afforded coumarins 2.

5,7-Dimethoxy-4-phenyl-2H-chromen-2-one (2a). Coumarin 2a was prepared from 1a (101.7 mg, $0.36 \mathrm{mmol}), 1$ fluoro-2,4,6-trimethylpyridinium trifluoromethanesulfonate (130.7 mg, $0.43 \mathrm{mmol}), \mathrm{Cu}(\mathrm{OAc})_{2}(3.3 \mathrm{mg}, 0.018 \mathrm{mmol})$, p-TsOH (69.1 mg, $0.36 \mathrm{mmol})$, and $\mathrm{PdCl}_{2}\left(\mathrm{CH}_{3} \mathrm{CN}\right)_{2}(4.7 \mathrm{mg}$, $0.018 \mathrm{mmol})$ in $\mathrm{HOAc}(1.4 \mathrm{~mL})$. The mixture was stirred at room temperature for $24 \mathrm{~h}$, and after the work-up, flash column chromatography (silica gel, hexane/EtOAc 8/2) afforded 2a (75 mg, 74\%) as a solid, whose data are coincidental with those reported: ${ }^{46} \mathrm{mp}\left(\mathrm{CH}_{2} \mathrm{Cl}_{2}\right)$ : 164-166 ${ }^{\circ} \mathrm{C}\left[\right.$ lit. ${ }^{46} \mathrm{mp}\left(\mathrm{CH}_{2} \mathrm{Cl}_{2} /\right.$ hexanes $) 164-165{ }^{\circ} \mathrm{C}$ ]; IR (ATR) $1713 \mathrm{~cm}^{-1}(\mathrm{C}=\mathrm{O}) ;{ }^{1} \mathrm{H}$ NMR $\left(\mathrm{CDCl}_{3}, 300 \mathrm{MHz}\right): \delta 3.42(\mathrm{~s}$, $\left.3 \mathrm{H}, \mathrm{OCH}_{3}\right), 3.87\left(\mathrm{~s}, 3 \mathrm{H}, \mathrm{OCH}_{3}\right), 6.01\left(\mathrm{~s}, 1 \mathrm{H}, \mathrm{H}_{3}\right), 6.23(\mathrm{~d}, J=$ $\left.2.3 \mathrm{~Hz}, 1 \mathrm{H}, \mathrm{H}_{6}\right), 6.53\left(\mathrm{~d}, J=2.3 \mathrm{~Hz}, 1 \mathrm{H}, \mathrm{H}_{8}\right), 7.22-7.30(\mathrm{~m}$, $\left.2 \mathrm{H}, \mathrm{H}_{3^{\prime}}, \mathrm{H}_{5^{\prime}}\right), 7.32-7.41\left(\mathrm{~m}, 3 \mathrm{H}, \mathrm{H}_{2^{\prime}}, \mathrm{H}_{4^{\prime}}, \mathrm{H}_{6^{\prime}}\right) ;{ }^{13} \mathrm{C}\left\{{ }^{1} \mathrm{H}\right\}$ NMR $\left(\mathrm{CDCl}_{3}, 75.5 \mathrm{MHz}\right): \delta 55.4\left(\mathrm{OCH}_{3}\right), 55.8\left(\mathrm{OCH}_{3}\right)$, 93.6 $\left(\mathrm{C}_{8}\right), 95.8\left(\mathrm{C}_{6}\right), 103.6\left(\mathrm{C}_{4 \mathrm{a}}\right), 112.7\left(\mathrm{C}_{3}\right), 127.1\left(\mathrm{C}_{2^{\prime}}, \mathrm{C}_{6^{\prime}}\right)$, $127.3\left(\mathrm{C}_{3^{\prime}}, \mathrm{C}_{5^{\prime}}\right), 127.9\left(\mathrm{C}_{4^{\prime}}\right), 139.8\left(\mathrm{C}_{1^{\prime}}\right), 155.7\left(\mathrm{C}_{4}\right), 157.2$ $\left(\mathrm{C}_{8 \mathrm{a}}\right), 158.2,163.4\left(\mathrm{C}_{5}, \mathrm{C}_{7}\right), 160.9(\mathrm{CO})$; MS (CI) $\mathrm{m} / z$ (rel intensity) $284\left(\mathrm{MH}^{+}+1,18\right), 283\left(\mathrm{MH}^{+}, 100\right), 282(34), 254$ (7); HRMS (CI) Calcd. for $\mathrm{C}_{17} \mathrm{H}_{15} \mathrm{O}_{4}\left[\mathrm{MH}^{+}\right]$: 283.0965; found, 283.0958.

5,7-Dimethoxy-4-(4-methoxyphenyl)-2H-chromen-2-one (2b). Coumarin $\mathbf{2 b}$ was prepared from $\mathbf{1 b}(117.6 \mathrm{mg}, 0.37$ mmol), 1-fluoro-2,4,6-trimethylpyridinium trifluoromethanesulfonate (131.2 mg, $0.45 \mathrm{mmol}), \mathrm{Cu}(\mathrm{OAc})_{2}(3.5 \mathrm{mg}, 0.019$ $\mathrm{mmol})$, $p$ - TsOH $(72.2 \mathrm{mg}, 0.37 \mathrm{mmol})$, and $\mathrm{PdCl}_{2}\left(\mathrm{CH}_{3} \mathrm{CN}\right)_{2}$ $(4.9 \mathrm{mg}, 0.019 \mathrm{mmol})$ in HOAc $(1.5 \mathrm{~mL})$. The mixture was stirred at room temperature for $6 \mathrm{~h}$, and after the work-up, flash column chromatography (silica gel, hexane/EtOAc 7/3) afforded $\mathbf{2 b}(85.5 \mathrm{mg}, 73 \%)$ as a solid, whose data are coincidental with those reported: ${ }^{70} \mathrm{mp}\left(\mathrm{CH}_{2} \mathrm{Cl}_{2}\right): 153-155$ ${ }^{\circ} \mathrm{C}$ [lit. ${ }^{70} \mathrm{mp}$ (methanol) $\left.151-152{ }^{\circ} \mathrm{C}\right]$; IR (ATR) $1716 \mathrm{~cm}^{-1}$ $(\mathrm{C}=\mathrm{O}) ;{ }^{1} \mathrm{H}$ NMR $\left(\mathrm{CDCl}_{3}, 300 \mathrm{MHz}\right): \delta 3.46(\mathrm{~s}, 3 \mathrm{H}$, $\left.\mathrm{OCH}_{3}\right), 3.84\left(\mathrm{~s}, 6 \mathrm{H}, 2 \times \mathrm{OCH}_{3}\right), 5.95\left(\mathrm{~s}, 1 \mathrm{H}, \mathrm{H}_{3}\right), 6.22(\mathrm{~d}, J=$ $\left.2.4 \mathrm{~Hz}, 1 \mathrm{H}, \mathrm{H}_{6}\right), 6.48\left(\mathrm{~d}, J=2.4 \mathrm{~Hz}, 1 \mathrm{H}, \mathrm{H}_{8}\right), 6.88(\mathrm{~d}, J=8.8$ $\left.\mathrm{Hz}, 2 \mathrm{H}, \mathrm{H}_{3^{\prime}}, \mathrm{H}_{5^{\prime}}\right), 7.19\left(\mathrm{~d}, J=8.8 \mathrm{~Hz}, 2 \mathrm{H}, \mathrm{H}_{2^{\prime}}, \mathrm{H}_{6^{\prime}}\right) ;{ }^{13} \mathrm{C}\left\{{ }^{1} \mathrm{H}\right\}$ NMR $\left(\mathrm{CDCl}_{3}, 75.5 \mathrm{MHz}\right): \delta 55.3\left(\mathrm{OCH}_{3}\right), 55.4\left(\mathrm{OCH}_{3}\right)$, $55.7\left(\mathrm{OCH}_{3}\right), 93.6\left(\mathrm{C}_{8}\right), 95.7\left(\mathrm{C}_{6}\right), 103.6\left(\mathrm{C}_{4 \mathrm{a}}\right), 112.5\left(\mathrm{C}_{3}\right)$, $112.7\left(\mathrm{C}_{3^{\prime}}, \mathrm{C}_{5^{\prime}}\right), 128.7\left(\mathrm{C}_{2^{\prime}}, \mathrm{C}_{6^{\prime}}\right), 132.0\left(\mathrm{C}_{1^{\prime}}\right), 155.5\left(\mathrm{C}_{4}\right)$, $157.2\left(\mathrm{C}_{8 \mathrm{a}}\right), 158.3\left(\mathrm{C}_{4^{\prime}}\right), 159.6,163.3\left(\mathrm{C}_{5}, \mathrm{C}_{7}\right), 160.9(\mathrm{CO})$; MS (CI) $m / z$ (rel intensity) $314\left(\mathrm{MH}^{+}+1,20\right), 313\left(\mathrm{MH}^{+}\right.$, 100), 312 (60), 284 (18); HRMS (CI) Calcd. for $\mathrm{C}_{18} \mathrm{H}_{17} \mathrm{O}_{5}$ $\left[\mathrm{MH}^{+}\right]$: 313.1071; found, 313.1061.

5,7-Dimethoxy-4-(p-tolyl)-2H-chromen-2-one (2c). Coumarin $2 \mathrm{c}$ was prepared from $1 \mathrm{c}(105.9 \mathrm{mg}, 0.36 \mathrm{mmol}), 1-$ fluoro-2,4,6-trimethylpyridinium trifluoromethanesulfonate $(123.2 \mathrm{mg}, 0.43 \mathrm{mmol}), \mathrm{Cu}(\mathrm{OAc})_{2}(3.2 \mathrm{mg}, 0.018 \mathrm{mmol})$, p-TsOH (67.5 mg, $0.36 \mathrm{mmol})$, and $\mathrm{PdCl}_{2}\left(\mathrm{CH}_{3} \mathrm{CN}\right)_{2}(4.6 \mathrm{mg}$, $0.018 \mathrm{mmol})$ in HOAc $(1.4 \mathrm{~mL})$. The mixture was stirred at room temperature for $24 \mathrm{~h}$, and after the work-up, flash column chromatography (silica gel, petroleum ether/EtOAc $\mathrm{t}$ $8 / 2$ ) afforded $2 \mathrm{c}(75.9 \mathrm{mg}, 72 \%)$ as a solid, whose data are coincidental with those reported: ${ }^{70} \mathrm{mp}\left(\mathrm{CH}_{2} \mathrm{Cl}_{2}\right): 131-133$ ${ }^{\circ} \mathrm{C}\left[\right.$ lit. $^{70} \mathrm{mp}$ (ethanol/water) $\left.131-133{ }^{\circ} \mathrm{C}\right]$; IR (ATR) 1712 $\mathrm{cm}^{-1}(\mathrm{C}=\mathrm{O}) ;{ }^{1} \mathrm{H} \mathrm{NMR}\left(\mathrm{CDCl}_{3}, 300 \mathrm{MHz}\right): \delta 2.42(\mathrm{~s}, 3 \mathrm{H}$, $\left.\mathrm{CH}_{3}\right), 3.47\left(\mathrm{~s}, 3 \mathrm{H}, \mathrm{OCH}_{3}\right), 3.88\left(\mathrm{~s}, 3 \mathrm{H}, \mathrm{OCH}_{3}\right), 6.00(\mathrm{~s}, 1 \mathrm{H}$, $\left.\mathrm{H}_{3}\right), 6.25\left(\mathrm{~d}, J=2.4 \mathrm{~Hz}, 1 \mathrm{H}, \mathrm{H}_{6}\right), 6.53\left(\mathrm{~d}, J=2.4 \mathrm{~Hz}, 1 \mathrm{H}, \mathrm{H}_{8}\right)$, 7.15-7.21 (m, 4H, $\left.\mathrm{H}_{2^{\prime}}, \mathrm{H}_{3^{\prime}}, \mathrm{H}_{5^{\prime}}, \mathrm{H}_{6^{\prime}}\right) ;{ }^{13} \mathrm{C}\left\{{ }^{1} \mathrm{H}\right\} \mathrm{NMR}\left(\mathrm{CDCl}_{3}\right.$, $75.5 \mathrm{MHz}): \delta 21.3\left(\mathrm{CH}_{3}\right), 55.4\left(\mathrm{OCH}_{3}\right), 55.8\left(\mathrm{OCH}_{3}\right), 93.6$ $\left(\mathrm{C}_{8}\right), 95.8\left(\mathrm{C}_{6}\right), 103.6\left(\mathrm{C}_{4 \mathrm{a}}\right), 112.7\left(\mathrm{C}_{3}\right), 127.2\left(\mathrm{C}_{2^{\prime}}, \mathrm{C}_{6^{\prime}}\right)$, $128.0\left(\mathrm{C}_{3^{\prime}}, \mathrm{C}_{5^{\prime}}\right), 136.9\left(\mathrm{C}_{4^{\prime}}\right), 137.8\left(\mathrm{C}_{1^{\prime}}\right), 155.8\left(\mathrm{C}_{4}\right), 157.2$ $\left(\mathrm{C}_{8 \mathrm{a}}\right), 158.3,161.0\left(\mathrm{C}_{5}, \mathrm{C}_{7}\right), 163.3(\mathrm{CO}) ; \mathrm{MS}\left(\mathrm{ESI}^{+}\right) \mathrm{m} / z$ (rel intensity) $\left(\mathrm{MH}^{+}+1,15\right), 297\left(\mathrm{MH}^{+}, 100\right)$; HRMS $\left(\mathrm{ESI}^{+}\right)$ Calcd. for $\mathrm{C}_{18} \mathrm{H}_{17} \mathrm{O}_{4}\left[\mathrm{MH}^{+}\right]$: 297.1127; found, 297.1132.

4-[4-(tert-Butyl)phenyl]-5,7-dimethoxy-2H-chromen-2one $(2 d)$. Coumarin $2 \mathrm{~d}$ was prepared from $1 \mathrm{~d}(122.0 \mathrm{mg}, 0.36$ $\mathrm{mmol})$, 1-fluoro-2,4,6-trimethylpyridinium trifluoromethanesulfonate $(124.4 \mathrm{mg}, 0.43 \mathrm{mmol}), \mathrm{Cu}(\mathrm{OAc})_{2}(3.3 \mathrm{mg}, 0.018$ $\mathrm{mmol}$ ), $p$-TsOH $(68.2 \mathrm{mg}, 0.36 \mathrm{mmol})$, and $\mathrm{PdCl}_{2}\left(\mathrm{CH}_{3} \mathrm{CN}\right)_{2}$ $(4.7 \mathrm{mg}, 0.018 \mathrm{mmol})$ in HOAc $(1.4 \mathrm{~mL})$. The mixture was stirred at room temperature for $24 \mathrm{~h}$, and after the work-up, flash column chromatography (silica gel, petroleum ether/ EtOAc 8/2) afforded $2 \mathrm{~d}(101.0 \mathrm{mg}, 83 \%)$ as a solid: $\mathrm{mp}$ $\left(\mathrm{CH}_{2} \mathrm{Cl}_{2}\right): 176-178{ }^{\circ} \mathrm{C}$; IR (ATR) $1718 \mathrm{~cm}^{-1}(\mathrm{C}=\mathrm{O}) ;{ }^{1} \mathrm{H}$ NMR $\left(\mathrm{CDCl}_{3}, 300 \mathrm{MHz}\right): \delta 1.38\left(\mathrm{~s}, 9 \mathrm{H}, \mathrm{C}\left(\mathrm{CH}_{3}\right)_{3}\right), 3.43(\mathrm{~s}$, $\left.3 \mathrm{H}, \mathrm{OCH}_{3}\right), 3.87\left(\mathrm{~s}, 3 \mathrm{H}, \mathrm{OCH}_{3}\right), 6.02\left(\mathrm{~s}, 1 \mathrm{H}, \mathrm{H}_{3}\right), 6.25(\mathrm{~d}, J=$ $\left.2.4 \mathrm{~Hz}, 1 \mathrm{H}, \mathrm{H}_{6}\right), 6.53\left(\mathrm{~d}, J=2.4 \mathrm{~Hz}, 1 \mathrm{H}, \mathrm{H}_{8}\right), 7.19-7.22(\mathrm{~m}$, $\left.2 \mathrm{H}, \mathrm{H}_{3^{\prime}}, \mathrm{H}_{5^{\prime}}\right), 7.39-7.42\left(\mathrm{~m}, 2 \mathrm{H}, \mathrm{H}_{2^{\prime}}, \mathrm{H}_{6^{\prime}}\right) ;{ }^{13} \mathrm{C}\left\{{ }^{1} \mathrm{H}\right\} \mathrm{NMR}$ $\left(\mathrm{CDCl}_{3}, 75.5 \mathrm{MHz}\right): \delta 31.3\left(\mathrm{C}\left(\mathrm{CH}_{3}\right)_{3}\right), 34.7\left(\mathrm{C}\left(\mathrm{CH}_{3}\right)_{3}\right), 55.5$ $\left(\mathrm{OCH}_{3}\right), 55.8\left(\mathrm{OCH}_{3}\right), 93.6\left(\mathrm{C}_{8}\right), 95.8\left(\mathrm{C}_{6}\right), 103.7\left(\mathrm{C}_{4 \mathrm{a}}\right)$, 
$112.5\left(\mathrm{C}_{3}\right), 124.2\left(\mathrm{C}_{2^{\prime}}, \mathrm{C}_{6^{\prime}}\right), 127.0\left(\mathrm{C}_{3^{\prime}}, \mathrm{C}_{5^{\prime}}\right), 136.8\left(\mathrm{C}_{4^{\prime}}\right)$, $151.1\left(\mathrm{C}_{1^{\prime}}\right), 155.8\left(\mathrm{C}_{4}\right), 157.2\left(\mathrm{C}_{8 \mathrm{a}}\right), 158.3,161.0\left(\mathrm{C}_{5}, \mathrm{C}_{7}\right)$, $163.3(\mathrm{CO}) ; \mathrm{MS}\left(\mathrm{ESI}^{+}\right) \mathrm{m} / z$ (rel intensity) $340\left(\mathrm{MH}^{+}+1\right.$, 17), $339\left(\mathrm{MH}^{+}, 100\right)$; HRMS $\left(\mathrm{ESI}^{+}\right)$Calcd. for $\mathrm{C}_{21} \mathrm{H}_{23} \mathrm{O}_{4}$ $\left[\mathrm{MH}^{+}\right]:$339.1596; found, 339.1600.

4-(3,5-Dimethoxyphenyl)-5,7-dimethoxy-2H-chromen-2one (2e). Coumarin $2 \mathrm{e}$ was prepared from $1 \mathrm{e}(115.6 \mathrm{mg}, 0.34$ $\mathrm{mmol}$ ), 1-fluoro-2,4,6-trimethylpyridinium trifluoromethanesulfonate $(116.5 \mathrm{mg}, 0.40 \mathrm{mmol}), \mathrm{Cu}(\mathrm{OAc})_{2}(3.0 \mathrm{mg}, 0.017$ $\mathrm{mmol})$, $p$-TsOH $(63.9 \mathrm{mg}, 0.34 \mathrm{mmol})$, and $\mathrm{PdCl}_{2}\left(\mathrm{CH}_{3} \mathrm{CN}\right)_{2}$ $(8.6 \mathrm{mg}, 0.034 \mathrm{mmol})$ in $\mathrm{HOAc}(1.4 \mathrm{~mL})$. The mixture was stirred at room temperature for $24 \mathrm{~h}$, and after the work-up, flash column chromatography (silica gel, petroleum ether/ EtOAc 8/2) afforded 2e (63.9 $\mathrm{mg}, 55 \%, 79 \%$ conversion) as a solid: $\mathrm{mp}\left(\mathrm{CH}_{2} \mathrm{Cl}_{2}\right)$ : $126-128{ }^{\circ} \mathrm{C}$; IR (ATR) $1718 \mathrm{~cm}^{-1}(\mathrm{C}=$ $\mathrm{O}) ;{ }^{1} \mathrm{H} \mathrm{NMR}\left(\mathrm{CDCl}_{3}, 300 \mathrm{MHz}\right): \delta 3.49\left(\mathrm{~s}, 3 \mathrm{H}, \mathrm{OCH}_{3}\right), 3.81$ $\left(\mathrm{s}, 6 \mathrm{H}, 2 \times \mathrm{OCH}_{3}\right), 3.87\left(\mathrm{~s}, 3 \mathrm{H}, \mathrm{OCH}_{3}\right), 6.02\left(\mathrm{~s}, 1 \mathrm{H}, \mathrm{H}_{3}\right)$, $6.25\left(\mathrm{~d}, J=2.4 \mathrm{~Hz}, 1 \mathrm{H}, \mathrm{H}_{6}\right), 6.41\left(\mathrm{~d}, J=2.3 \mathrm{~Hz}, 2 \mathrm{H}, \mathrm{H}_{2^{\prime}}, \mathrm{H}_{6^{\prime}}\right)$, $6.49\left(\mathrm{t}, J=2.3 \mathrm{~Hz}, 1 \mathrm{H}, \mathrm{H}_{4^{\prime}}\right), 6.52\left(\mathrm{~d}, J=2.4 \mathrm{~Hz}, 1 \mathrm{H}, \mathrm{H}_{8}\right)$; ${ }^{13} \mathrm{C}\left\{{ }^{1} \mathrm{H}\right\}$ NMR $\left(\mathrm{CDCl}_{3}, 75.5 \mathrm{MHz}\right): \delta 55.5\left(2 \times \mathrm{OCH}_{3}\right), 55.6$ $\left(\mathrm{OCH}_{3}\right), 55.8\left(\mathrm{OCH}_{3}\right), 93.6\left(\mathrm{C}_{8}\right), 95.9\left(\mathrm{C}_{6}\right), 100.0\left(\mathrm{C}_{4^{\prime}}\right)$, $103.5\left(\mathrm{C}_{4 \mathrm{a}}\right), 105.4\left(\mathrm{C}_{2^{\prime}}, \mathrm{C}_{6^{\prime}}\right), 112.4\left(\mathrm{C}_{3}\right), 141.7\left(\mathrm{C}_{1^{\prime}}\right), 155.5$ $\left(\mathrm{C}_{4}\right), 157.1\left(\mathrm{C}_{8 \mathrm{a}}\right), 158.2\left(\mathrm{C}_{7}\right), 160.0\left(\mathrm{C}_{3^{\prime}}, \mathrm{C}_{5^{\prime}}\right), 160.9\left(\mathrm{C}_{5}\right)$, 163.4 (CO); MS $\left(\mathrm{ESI}^{+}\right) \mathrm{m} / z$ (rel intensity) $344\left(\mathrm{MH}^{+}+1\right.$, 16), $343\left(\mathrm{MH}^{+}, 100\right)$; HRMS $\left(\mathrm{ESI}^{+}\right)$Calcd. for $\mathrm{C}_{19} \mathrm{H}_{19} \mathrm{O}_{6}$ $\left[\mathrm{MH}^{+}\right]$: 343.1182; found, 343.1180.

4-(Furan-3-yl)-5,7-dimethoxy-2H-chromen-2-one (2f). Coumarin $2 \mathrm{f}$ was prepared from $1 \mathrm{f}(98.1 \mathrm{mg}, 0.36 \mathrm{mmol})$, 1-fluoro-2,4,6-trimethylpyridinium trifluoromethanesulfonate (124.1 mg, $0.43 \mathrm{mmol}), \mathrm{Cu}(\mathrm{OAc})_{2}(3.2 \mathrm{mg}, 0.018 \mathrm{mmol})$, p-TsOH (68.0 mg, $0.36 \mathrm{mmol})$, and $\mathrm{PdCl}_{2}\left(\mathrm{CH}_{3} \mathrm{CN}\right)_{2}(4.6 \mathrm{mg}$, $0.018 \mathrm{mmol})$ in $\mathrm{HOAc}(1.4 \mathrm{~mL})$. The mixture was stirred at room temperature for $24 \mathrm{~h}$, and after the work-up, flash column chromatography (silica gel, petroleum ether/EtOAc 8/ 2) afforded $2 \mathrm{f}(20.6 \mathrm{mg}, 21 \%, 75 \%$ conversion $)$ as a solid: $\mathrm{mp}$ $\left(\mathrm{CH}_{2} \mathrm{Cl}_{2}\right): 153-155^{\circ} \mathrm{C}$; IR (ATR) $1714 \mathrm{~cm}^{-1}(\mathrm{C}=\mathrm{O}) ;{ }^{1} \mathrm{H}$ NMR $\left(\mathrm{CDCl}_{3}, 300 \mathrm{MHz}\right): \delta 3.67\left(\mathrm{~s}, 3 \mathrm{H}, \mathrm{OCH}_{3}\right), 3.89(\mathrm{~s}, 3 \mathrm{H}$, $\left.\mathrm{OCH}_{3}\right), 6.09\left(\mathrm{~s}, 1 \mathrm{H}, \mathrm{H}_{3}\right), 6.30\left(\mathrm{~d}, J=2.4 \mathrm{~Hz}, 1 \mathrm{H}, \mathrm{H}_{6}\right), 6.49-$ $6.52\left(\mathrm{~m}, 2 \mathrm{H}, \mathrm{H}_{8}, \mathrm{H}_{4^{\prime}}\right), 7.45\left(\mathrm{t}, J=1.8 \mathrm{~Hz}, 1 \mathrm{H}, \mathrm{H}_{5^{\prime}}\right), 7.56-7.57$ $\left(\mathrm{m}, 1 \mathrm{H}, \mathrm{H}_{2^{\prime}}\right) ;{ }^{13} \mathrm{C}\left\{{ }^{1} \mathrm{H}\right\}$ NMR $\left(\mathrm{CDCl}_{3}, 75.5 \mathrm{MHz}\right): \delta 55.3$ $\left(\mathrm{OCH}_{3}\right), 55.8\left(\mathrm{OCH}_{3}\right), 93.6\left(\mathrm{C}_{8}\right), 95.7\left(\mathrm{C}_{6}\right), 103.4\left(\mathrm{C}_{4 \mathrm{a}}\right)$, $112.1\left(\mathrm{C}_{4^{\prime}}\right), 112.5\left(\mathrm{C}_{3}\right), 125.0\left(\mathrm{C}_{3^{\prime}}\right), 140.3\left(\mathrm{C}_{2^{\prime}}\right), 142.0\left(\mathrm{C}_{5^{\prime}}\right)$, $146.7\left(\mathrm{C}_{4}\right), 157.3,158.2,160.8\left(\mathrm{C}_{8 \mathrm{a}}, \mathrm{C}_{5}, \mathrm{C}_{7}\right), 163.3(\mathrm{CO})$; $\mathrm{MS}$ $\left(\mathrm{ESI}^{+}\right) \mathrm{m} / z$ (rel intensity) $274\left(\mathrm{MH}^{+}+1,12\right), 273\left(\mathrm{MH}^{+}\right.$, 100); HRMS (ESI ${ }^{+}$) Calcd. for $\mathrm{C}_{15} \mathrm{H}_{13} \mathrm{O}_{5}\left[\mathrm{MH}^{+}\right]$: 273.0763; found, 273.0769 .

4-(3,5-Difluorophenyl)-5,7-dimethoxy-2H-chromen-2-one (2g). Coumarin $2 \mathrm{~g}$ was prepared from $1 \mathrm{~g}$ (87.5 mg, 0.27 $\mathrm{mmol}$ ), 1-fluoro-2,4,6-trimethylpyridinium trifluoromethanesulfonate $(94.9 \mathrm{mg}, 0.33 \mathrm{mmol}), \mathrm{Cu}(\mathrm{OAc})_{2}(3.0 \mathrm{mg}, 0.016$ mmol), $p$ - TsOH (79.3 mg, $0.27 \mathrm{mmol})$, and $\mathrm{PdCl}_{2}\left(\mathrm{CH}_{3} \mathrm{CN}\right)_{2}$ $(8.5 \mathrm{mg}, 0.032 \mathrm{mmol})$ in mesitylene $(1.1 \mathrm{~mL})$. The mixture was stirred at $70{ }^{\circ} \mathrm{C}$ for $24 \mathrm{~h}$, and after the work-up, flash column chromatography (silica gel, hexane/EtOAc 8/2) afforded $2 \mathrm{~g}(25.8 \mathrm{mg}, 30 \%)$ as a solid: $\mathrm{mp}\left(\mathrm{CH}_{2} \mathrm{Cl}_{2}\right): 170-$ $172{ }^{\circ} \mathrm{C}$; IR (ATR) $1724 \mathrm{~cm}^{-1}(\mathrm{C}=\mathrm{O}) ;{ }^{1} \mathrm{H} \mathrm{NMR}\left(\mathrm{CDCl}_{3}, 300\right.$ $\mathrm{MHz}): \delta 3.48\left(\mathrm{~s}, 3 \mathrm{H}, \mathrm{OCH}_{3}\right), 3.87\left(\mathrm{~s}, 3 \mathrm{H}, \mathrm{OCH}_{3}\right), 5.97(\mathrm{~s}$, $\left.1 \mathrm{H}, \mathrm{H}_{3}\right), 6.23\left(\mathrm{~d}, J=2.4 \mathrm{~Hz}, 1 \mathrm{H}, \mathrm{H}_{6}\right), 6.51(\mathrm{~d}, J=2.4 \mathrm{~Hz}, 1 \mathrm{H}$, $\left.\mathrm{H}_{8}\right)$, 6.72-6.91 (m, 3H, $\left.\mathrm{H}_{2^{\prime}}, \mathrm{H}_{4^{\prime}}, \mathrm{H}_{6^{\prime}}\right) ;{ }^{13} \mathrm{C}\left\{{ }^{1} \mathrm{H}\right\}$ NMR $\left(\mathrm{CDCl}_{3}, 75.5 \mathrm{MHz}\right): \delta 55.6\left(\mathrm{OCH}_{3}\right), 55.8\left(\mathrm{OCH}_{3}\right), 93.7\left(\mathrm{C}_{8}\right)$, $95.8\left(\mathrm{C}_{6}\right), 102.8\left(\mathrm{C}_{4 \mathrm{a}}\right), 103.2\left(\mathrm{t}, \mathrm{J}=25.2 \mathrm{~Hz}, \mathrm{C}_{4^{\prime}}\right), 110.9-$ $112.7\left(\mathrm{~m}, \mathrm{C}_{2^{\prime}}, \mathrm{C}_{6^{\prime}}\right), 112.7\left(\mathrm{C}_{3}\right), 142.8\left(\mathrm{t}, J=10.1 \mathrm{~Hz}, \mathrm{C}_{1^{\prime}}\right)$, $153.0\left(\mathrm{C}_{4}\right), 157.1\left(\mathrm{C}_{8 \mathrm{a}}\right), 157.9\left(\mathrm{C}_{5}\right), 160.3\left(\mathrm{C}_{7}\right), 162.2(\mathrm{dd}, J=$
248.1, $13.2 \mathrm{~Hz}, \mathrm{C}_{3^{\prime}}, \mathrm{C}_{5^{\prime}}$ ), 163.8 (CO); MS (EI) $\mathrm{m} / z$ (rel intensity) $319.1\left(\mathrm{M}^{+}+1,19\right), 318.1\left(\mathrm{M}^{+}, 100\right), 291.1(16)$, 290.1 (93), 275.1 (39), 247 (12), 188 (14), 175 (15), 69 (10); HRMS (CI) Calcd. for $\mathrm{C}_{17} \mathrm{H}_{13} \mathrm{~F}_{2} \mathrm{O}_{4}\left[\mathrm{MH}^{+}\right]$: 319.0776; found, 319.0770 .

5,7-Dimethoxy-2H-chromen-2-one ( $2 h$ ). Coumarin $2 \mathrm{~h}$ was prepared from $\mathbf{l h}$ (94.4 mg, $0.45 \mathrm{mmol})$, 1-fluoro-2,4,6trimethylpyridinium trifluoromethanesulfonate $(157.4 \mathrm{mg}$, $0.54 \mathrm{mmol}), \mathrm{Cu}(\mathrm{OAc})_{2}(4.1 \mathrm{mg}, 0.023 \mathrm{mmol}), p-\mathrm{TsOH}$ (131.6 mg, $0.45 \mathrm{mmol})$, and $\mathrm{PdCl}_{2}\left(\mathrm{CH}_{3} \mathrm{CN}\right)_{2}(5.9 \mathrm{mg}, 0.023$ $\mathrm{mmol})$ in mesitylene $(1.8 \mathrm{~mL})$. The mixture was stirred at 70 ${ }^{\circ} \mathrm{C}$ for $4 \mathrm{~h}$, and after the work-up, flash column chromatography (silica gel, hexane/EtOAc 7/3) afforded $\mathbf{2 h}$ $(64.9 \mathrm{mg}, 69 \%)$ as a white solid, whose data are coincidental with those reported: ${ }^{46} \mathrm{mp}\left(\mathrm{CH}_{2} \mathrm{Cl}_{2}\right): 144-146{ }^{\circ} \mathrm{C}\left[\right.$ lit. ${ }^{46} \mathrm{mp}$ $\left(\mathrm{CH}_{2} \mathrm{Cl}_{2} /\right.$ pentanes) $\left.143-145{ }^{\circ} \mathrm{C}\right]$; IR (ATR) $1724 \mathrm{~cm}^{-1}$ $(\mathrm{C}=\mathrm{O}) ;{ }^{1} \mathrm{H}$ NMR $\left(\mathrm{CDCl}_{3}, 300 \mathrm{MHz}\right): \delta 3.82(\mathrm{~s}, 3 \mathrm{H}$, $\left.\mathrm{OCH}_{3}\right), 3.89\left(\mathrm{~s}, 3 \mathrm{H}, \mathrm{OCH}_{3}\right), 6.15\left(\mathrm{~d}, J=9.7 \mathrm{~Hz}, 1 \mathrm{H}, \mathrm{H}_{3}\right)$, $6.28\left(\mathrm{~d}, J=2.1 \mathrm{~Hz}, 1 \mathrm{H}, \mathrm{H}_{6}\right), 6.41\left(\mathrm{~d}, J=2.1 \mathrm{~Hz}, 1 \mathrm{H}, \mathrm{H}_{8}\right), 7.96$ $\left(\mathrm{d}, J=9.7 \mathrm{~Hz}, 1 \mathrm{H}, \mathrm{H}_{4}\right) ;{ }^{13} \mathrm{C}\left\{{ }^{1} \mathrm{H}\right\} \mathrm{NMR}\left(\mathrm{CDCl}_{3}, 75.5 \mathrm{MHz}\right): \delta$ $55.8\left(\mathrm{OCH}_{3}\right), 55.9\left(\mathrm{OCH}_{3}\right), 92.8\left(\mathrm{C}_{8}\right), 94.8\left(\mathrm{C}_{6}\right), 104.0$ $\left(\mathrm{C}_{4 \mathrm{a}}\right), 110.9\left(\mathrm{C}_{3}\right), 138.7\left(\mathrm{C}_{4}\right), 156.8\left(\mathrm{C}_{8 \mathrm{a}}\right), 157.0,163.7\left(\mathrm{C}_{5}\right.$, $\mathrm{C}_{7}$ ), 161.5 (CO); MS (EI) $\mathrm{m} / z$ (rel intensity) $207\left(\mathrm{M}^{+}+1\right.$, 12), $206\left(\mathrm{M}^{+}, 100\right), 178$ (79), 163 (48), 135 (22); HRMS (CI) Calcd. for $\mathrm{C}_{11} \mathrm{H}_{11} \mathrm{O}_{4}\left[\mathrm{MH}^{+}\right]$: 207.0652; found, 207.0658.

5,7-Dimethoxy-4-methyl-2H-chromen-2-one (2i). Coumarin $2 \mathbf{i}$ was prepared from $\mathbf{1 i}(84.2 \mathrm{mg}, 0.38 \mathrm{mmol}), 1-$ fluoro-2,4,6-trimethylpyridinium trifluoromethanesulfonate (131.5 mg, $0.45 \mathrm{mmol}), \mathrm{Cu}(\mathrm{OAc})_{2}(3.4 \mathrm{mg}, 0.018 \mathrm{mmol})$, p-TsOH $(72.1 \mathrm{mg}, 0.38 \mathrm{mmol})$, and $\mathrm{PdCl}_{2}\left(\mathrm{CH}_{3} \mathrm{CN}\right)_{2}(9.8 \mathrm{mg}$, $0.036 \mathrm{mmol})$ in mesitylene $(1.5 \mathrm{~mL})$. The mixture was stirred at $70{ }^{\circ} \mathrm{C}$ for $24 \mathrm{~h}$, and after the work-up, flash column chromatography (silica gel, hexane/EtOAc 7/3) afforded $\mathbf{2 i}$ $(50.3 \mathrm{mg}, 60 \%)$ as a white solid, whose data are coincidental with those reported: ${ }^{46} \mathrm{mp}\left(\mathrm{CH}_{2} \mathrm{Cl}_{2}\right): 166-168{ }^{\circ} \mathrm{C}$ [lit. ${ }^{46} \mathrm{mp}$ (methanol): $168-170{ }^{\circ} \mathrm{C}$; IR (ATR) $1740 \mathrm{~cm}^{-1}(\mathrm{C}=\mathrm{O}) ;{ }^{1} \mathrm{H}$ NMR $\left(\mathrm{CDCl}_{3}, 300 \mathrm{MHz}\right): \delta 2.50\left(\mathrm{~d}, J=1.2 \mathrm{~Hz}, 3 \mathrm{H}, \mathrm{CH}_{3}\right)$, $3.82\left(\mathrm{~s}, 3 \mathrm{H}, \mathrm{OCH}_{3}\right), 3.83\left(\mathrm{~s}, 3 \mathrm{H}, \mathrm{OCH}_{3}\right), 5.91\left(\right.$ br s, $\left.1 \mathrm{H}, \mathrm{H}_{3}\right)$, $6.26\left(\mathrm{~d}, J=2.4 \mathrm{~Hz}, 1 \mathrm{H}, \mathrm{H}_{6}\right), 6.39\left(\mathrm{~d}, J=2.4 \mathrm{~Hz}, 1 \mathrm{H}, \mathrm{H}_{8}\right)$; ${ }^{13} \mathrm{C}\left\{{ }^{1} \mathrm{H}\right\}$ NMR $\left(\mathrm{CDCl}_{3}, 75.5 \mathrm{MHz}\right): \delta 24.2\left(\mathrm{CH}_{3}\right), 55.6$ $\left(\mathrm{OCH}_{3}\right), 55.7\left(\mathrm{OCH}_{3}\right), 93.4\left(\mathrm{C}_{8}\right), 95.4\left(\mathrm{C}_{6}\right), 104.8\left(\mathrm{C}_{4 \mathrm{a}}\right)$, $111.3\left(\mathrm{C}_{3}\right), 154.5\left(\mathrm{C}_{8 \mathrm{a}}\right), 156.9\left(\mathrm{C}_{4}\right), 159.1,162.8\left(\mathrm{C}_{5}, \mathrm{C}_{7}\right)$, 161.0 (CO); MS (EI) $m / z$ (rel intensity) $221\left(\mathrm{M}^{+}+1,13\right)$, 220 ( $\left.\mathrm{M}^{+}, 100\right), 193$ (11), 192 (91), 178 (10), 177 (73), 149 (13); HRMS (CI) Calcd. for $\mathrm{C}_{12} \mathrm{H}_{13} \mathrm{O}_{4}\left[\mathrm{MH}^{+}\right]$: 221.0808; found, 221.0811.

4-Ethyl-5,7-dimethoxy-2H-chromen-2-one (2j). Coumarin $2 \mathbf{j}$ was prepared from $\mathbf{1 j}(61.0 \mathrm{mg}, 0.26 \mathrm{mmol}), 1$-fluoro-2,4,6trimethylpyridinium trifluoromethanesulfonate $(94.3 \mathrm{mg}, 0.31$ $\mathrm{mmol}), \mathrm{Cu}(\mathrm{OAc})_{2}(2.4 \mathrm{mg}, 0.013 \mathrm{mmol}), p-\mathrm{TsOH}(49.9 \mathrm{mg}$, $0.26 \mathrm{mmol})$, and $\mathrm{PdCl}_{2}\left(\mathrm{CH}_{3} \mathrm{CN}\right)_{2}(6.8 \mathrm{mg}, 0.026 \mathrm{mmol})$ in mesitylene $(1 \mathrm{~mL})$. The mixture was stirred at $70{ }^{\circ} \mathrm{C}$ for $24 \mathrm{~h}$, and after the work-up, flash column chromatography (silica gel, hexane/EtOAc $7 / 3$ ) afforded $2 \mathbf{j}(29.8 \mathrm{mg}, 49 \%)$ as a solid, whose data are coincidental with those reported: ${ }^{71} \mathrm{mp}$ $\left(\mathrm{CH}_{2} \mathrm{Cl}_{2}\right)$ : 141-143 ${ }^{\circ} \mathrm{C}$ [lit. ${ }^{71} \mathrm{mp}$ (EtOH: $\mathrm{H}_{2} \mathrm{O}$ ) 146-148 $\left.{ }^{\circ} \mathrm{C}\right]$; IR (ATR) $1710 \mathrm{~cm}^{-1}(\mathrm{C}=\mathrm{O})$; ${ }^{1} \mathrm{H}$ NMR $\left(\mathrm{CDCl}_{3}, 300\right.$ $\mathrm{MHz}): \delta 1.21\left(\mathrm{t}, J=7.3 \mathrm{~Hz}, 3 \mathrm{H}, \mathrm{CH}_{3}\right), 2.94(\mathrm{q}, J=7.3 \mathrm{~Hz}$, $\left.2 \mathrm{H}, \mathrm{CH}_{2}\right), 3.84\left(\mathrm{~s}, 3 \mathrm{H}, \mathrm{OCH}_{3}\right), 3.87\left(\mathrm{~s}, 3 \mathrm{H}, \mathrm{OCH}_{3}\right), 6.00(\mathrm{~s}$, $\left.1 \mathrm{H}, \mathrm{H}_{3}\right), 6.30\left(\mathrm{~d}, J=2.4 \mathrm{~Hz}, 1 \mathrm{H}, \mathrm{H}_{6}\right), 6.45(\mathrm{~d}, J=2.4 \mathrm{~Hz}, 1 \mathrm{H}$, $\left.\mathrm{H}_{8}\right) ;{ }^{13} \mathrm{C}\left\{{ }^{1} \mathrm{H}\right\} \operatorname{NMR}\left(\mathrm{CDCl}_{3}, 75.5 \mathrm{MHz}\right): \delta 13.5\left(\mathrm{CH}_{3}\right), 29.4$ $\left(\mathrm{CH}_{2}\right)$, $55.7\left(\mathrm{OCH}_{3}\right), 55.8\left(\mathrm{OCH}_{3}\right), 93.6\left(\mathrm{C}_{8}\right), 95.6\left(\mathrm{C}_{6}\right)$, $104.3\left(\mathrm{C}_{4 \mathrm{a}}\right), 109.7\left(\mathrm{C}_{3}\right), 157.2\left(\mathrm{C}_{8 \mathrm{a}}\right), 158.8\left(\mathrm{C}_{4}\right), 159.9,162.6$ 
$\left(\mathrm{C}_{5}, \mathrm{C}_{7}\right), 161.4(\mathrm{CO})$; MS (EI) $m / z$ (rel intensity) $235\left(\mathrm{M}^{+}+\right.$ 1, 15), $234\left(\mathrm{M}^{+}, 100\right), 207$ (12), 206 (86), 205 (16), 191 (52), 161 (12), 103 (11); HRMS (CI) Calcd. for $\mathrm{C}_{13} \mathrm{H}_{15} \mathrm{O}_{4}\left[\mathrm{MH}^{+}\right]$: 235.0965; found, 235.0966 .

5,7-Dimethoxy-4-propyl-2H-chromen-2-one (2k). Coumarin $2 \mathbf{k}$ was prepared from $\mathbf{1 k}(77.0 \mathrm{mg}, 0.31 \mathrm{mmol}), 1-$ fluoro-2,4,6-trimethylpyridinium trifluoromethanesulfonate (112.4 mg, $0.37 \mathrm{mmol}), \mathrm{Cu}(\mathrm{OAc})_{2}(2.9 \mathrm{mg}, 0.015 \mathrm{mmol})$, p-TsOH (59.4 mg, $0.31 \mathrm{mmol})$, and $\mathrm{PdCl}_{2}\left(\mathrm{CH}_{3} \mathrm{CN}\right)_{2}(8.0 \mathrm{mg}$, $0.030 \mathrm{mmol})$ in mesitylene $(1.2 \mathrm{~mL})$. The mixture was stirred at $70{ }^{\circ} \mathrm{C}$ for $24 \mathrm{~h}$, and after the work-up, flash column chromatography (silica gel, hexane/EtOAc 7/3) afforded $2 \mathbf{k}$ $(37.9 \mathrm{mg}, 50 \%)$ as a solid, whose data are coincidental with those reported: ${ }^{72} \mathrm{mp}\left(\mathrm{CH}_{2} \mathrm{Cl}_{2}\right): 109-111{ }^{\circ} \mathrm{C}\left[\right.$ lit. $^{72} \mathrm{mp}$ $\left(\mathrm{CHCl}_{3}\right) 113.5-115^{\circ} \mathrm{C}$; IR (ATR) $1713 \mathrm{~cm}^{-1}(\mathrm{C}=\mathrm{O}) ;{ }^{1} \mathrm{H}$ NMR $\left(\mathrm{CDCl}_{3}, 300 \mathrm{MHz}\right): \delta 1.00\left(\mathrm{t}, J=7.3 \mathrm{~Hz}, 3 \mathrm{H}, \mathrm{CH}_{2}-\right.$ $\left.\mathrm{CH}_{2}-\mathrm{CH}_{3}\right), 1.52-1.69\left(\mathrm{~m}, 2 \mathrm{H}, \mathrm{CH}_{2}-\mathrm{CH}_{2}-\mathrm{CH}_{3}\right), 2.82-2.88$ $\left(\mathrm{m}, 2 \mathrm{H}, \mathrm{CH}_{2}-\mathrm{CH}_{2}-\mathrm{CH}_{3}\right), 3.84\left(\mathrm{~s}, 3 \mathrm{H}, \mathrm{OCH}_{3}\right), 3.86(\mathrm{~s}, 3 \mathrm{H}$, $\left.\mathrm{OCH}_{3}\right), 5.96\left(\mathrm{~s}, 1 \mathrm{H}, \mathrm{H}_{3}\right), 6.30\left(\mathrm{~d}, J=2.4 \mathrm{~Hz}, 1 \mathrm{H}, \mathrm{H}_{6}\right), 6.45(\mathrm{~d}$, $\left.J=2.4 \mathrm{~Hz}, 1 \mathrm{H}, \mathrm{H}_{8}\right) ;{ }^{13} \mathrm{C}\left\{{ }^{1} \mathrm{H}\right\} \mathrm{NMR}\left(\mathrm{CDCl}_{3}, 75.5 \mathrm{MHz}\right): \delta$ $14.0\left(\mathrm{CH}_{2}-\mathrm{CH}_{2}-\mathrm{CH}_{3}\right), 22.8\left(\mathrm{CH}_{2}-\mathrm{CH}_{2}-\mathrm{CH}_{3}\right), \quad 38.5$ $\left(\mathrm{CH}_{2}-\mathrm{CH}_{2}-\mathrm{CH}_{3}\right), 55.7\left(\mathrm{OCH}_{3}\right), 55.8\left(\mathrm{OCH}_{3}\right), 93.6\left(\mathrm{C}_{8}\right)$, 95.6 $\left(\mathrm{C}_{6}\right), 104.3\left(\mathrm{C}_{4 \mathrm{a}}\right), 110.7\left(\mathrm{C}_{3}\right), 157.3\left(\mathrm{C}_{8 \mathrm{a}}\right), 158.2\left(\mathrm{C}_{4}\right)$, 158.7, $162.6\left(\mathrm{C}_{5}, \mathrm{C}_{7}\right), 161.3(\mathrm{CO}) ; \mathrm{MS}(\mathrm{EI}) \mathrm{m} / \mathrm{z}$ (rel intensity) 249.1 $\left(\mathrm{M}^{+}+1,15\right), 248.1\left(\mathrm{M}^{+}, 100\right), 233.1$ (36), 220.1 (63), 205.1 (41), 192.1 (56), 191.1 (28), 178 (15), 177.1 (25), 161.1 (22), 146 (15), 91 (10), 77.1 (12), 69 (14); HRMS (CI) Calcd. for $\mathrm{C}_{14} \mathrm{H}_{17} \mathrm{O}_{4}\left[\mathrm{MH}^{+}\right]$: 249.1121; found, 249.1123.

6,7-Dimethoxy-4-phenyl-2H-chromen-2-one (2p). Coumarin $2 \mathrm{p}$ was prepared from $1 \mathrm{p}(60 \mathrm{mg}, 0.21 \mathrm{mmol}), 1-$ fluoro-2,4,6-trimethylpyridinium trifluoromethanesulfonate (75.9 mg, $0.25 \mathrm{mmol}), \mathrm{Cu}(\mathrm{OAc})_{2}(2.2 \mathrm{mg}, 0.010 \mathrm{mmol}), p$ $\mathrm{TsOH}(40.2 \mathrm{mg}, 0.21 \mathrm{mmol})$, and $\mathrm{PdCl}_{2}\left(\mathrm{CH}_{3} \mathrm{CN}\right)_{2}(5.6 \mathrm{mg}$, $0.021 \mathrm{mmol})$ in mesitylene $(1.0 \mathrm{~mL})$. The mixture was stirred at $70{ }^{\circ} \mathrm{C}$ for $24 \mathrm{~h}$, and after the work-up, flash column chromatography (silica gel, hexane/EtOAc 7/3) afforded $2 \mathbf{p}$ $(17.8 \mathrm{mg}, 30 \%)$ as an oil, whose data are coincidental with those reported: ${ }^{73}$ IR (ATR): $1715 \mathrm{~cm}^{-1}(\mathrm{C}=\mathrm{O}) ;{ }^{1} \mathrm{H}$ NMR $\left(\mathrm{CDCl}_{3}, 300 \mathrm{MHz}\right): \delta 3.77\left(\mathrm{~s}, 3 \mathrm{H}, \mathrm{OCH}_{3}\right), 3.99(\mathrm{~s}, 3 \mathrm{H}$, $\left.\mathrm{OCH}_{3}\right), 6.26\left(\mathrm{~s}, 1 \mathrm{H}, \mathrm{H}_{3}\right), 6.88\left(\mathrm{~s}, 1 \mathrm{H}, \mathrm{H}_{8}\right), 6.94\left(\mathrm{~s}, 1 \mathrm{H}, \mathrm{H}_{5}\right)$, 7.46-7.56 (m, 5H, Ph); ${ }^{13} \mathrm{C}\left\{{ }^{1} \mathrm{H}\right\}$ NMR $\left(\mathrm{CDCl}_{3}, 75.5 \mathrm{MHz}\right): \delta$ $56.3\left(\mathrm{OCH}_{3}\right), 56.4\left(\mathrm{OCH}_{3}\right), 100.3\left(\mathrm{C}_{8}\right), 107.5\left(\mathrm{C}_{5}\right), 111.4$ $\left(\mathrm{C}_{4 \mathrm{a}}\right), 112.4\left(\mathrm{C}_{3}\right), 128.3,128.9,129.7\left(\mathrm{C}_{2^{\prime}}, \mathrm{C}_{3^{\prime}}, \mathrm{C}_{4^{\prime}}, \mathrm{C}_{5^{\prime}}, \mathrm{C}_{6^{\prime}}\right)$, $135.8\left(\mathrm{C}_{1^{\prime}}\right), 146.1\left(\mathrm{C}_{4}\right), 150.2\left(\mathrm{C}_{8 \mathrm{a}}\right), 152.9,155.6\left(\mathrm{C}_{6}, \mathrm{C}_{7}\right)$, 161.4 (CO); MS (EI) $m / z$ (rel intensity): $282\left(\mathrm{M}^{+}, 100\right), 254$ (23), 239 (16), 207 (27), 168 (10), 155 (12), 139 (15), 127 (17); HRMS (ESI ${ }^{+}$) Calcd. for $\mathrm{C}_{17} \mathrm{H}_{15} \mathrm{O}_{4}\left[\mathrm{MH}^{+}\right]$: 283.0965; found, 283.0957.

6,7-Dimethoxy-4-methyl-2H-chromen-2-one (2q). Coumarin $2 \mathrm{q}$ was prepared from $\mathbf{1 q}(60.1 \mathrm{mg}, 0.27 \mathrm{mmol}), 1-$ fluoro-2,4,6-trimethylpyridinium trifluoromethanesulfonate (98.9 mg, $0.33 \mathrm{mmol}), \mathrm{Cu}(\mathrm{OAc})_{2}$ (5.1 mg, $\left.0.027 \mathrm{mmol}\right), p$ $\mathrm{TsOH}(52.2 \mathrm{mg}, 0.27 \mathrm{mmol})$, and $\mathrm{PdCl}_{2}\left(\mathrm{CH}_{3} \mathrm{CN}\right)_{2}(7.1 \mathrm{mg}$, $0.027 \mathrm{mmol})$ in mesitylene $(1.3 \mathrm{~mL})$. The mixture was stirred at $70{ }^{\circ} \mathrm{C}$ for $24 \mathrm{~h}$, and after the work-up, flash column chromatography (silica gel, hexane/EtOAc 7/3) afforded $\mathbf{2 q}$ (20.3 mg, 34\%) as a solid, whose data are coincidental with those reported: ${ }^{71} \mathrm{mp}\left(\mathrm{CH}_{2} \mathrm{Cl}_{2}\right): 128-129{ }^{\circ} \mathrm{C}\left[\right.$ lit. $^{71} \mathrm{mp}$ (EtOH: $\mathrm{H}_{2} \mathrm{O}$ ) $128-129{ }^{\circ} \mathrm{C}$ ]; IR (ATR): $1715 \mathrm{~cm}^{-1}$ (C=O); ${ }^{1} \mathrm{H} \mathrm{NMR}\left(\mathrm{CDCl}_{3}, 300 \mathrm{MHz}\right): \delta 2.41\left(\mathrm{~d}, J=1.0 \mathrm{~Hz}, 3 \mathrm{H}, \mathrm{CH}_{3}\right)$, $3.94\left(\mathrm{~s}, 6 \mathrm{H}, 2 \times \mathrm{OCH}_{3}\right), 6.17\left(\mathrm{~d}, J=1.0 \mathrm{~Hz}, 1 \mathrm{H}, \mathrm{H}_{3}\right), 6.85(\mathrm{~s}$, $\left.1 \mathrm{H}, \mathrm{H}_{5}\right), 6.94\left(\mathrm{~s}, 1 \mathrm{H}, \mathrm{H}_{8}\right) ;{ }^{13} \mathrm{C}\left\{{ }^{1} \mathrm{H}\right\} \mathrm{NMR}\left(\mathrm{CDCl}_{3}, 75.5\right.$
$\mathrm{MHz}): \delta 18.9\left(\mathrm{CH}_{3}\right), 56.3\left(\mathrm{OCH}_{3}\right), 56.4\left(\mathrm{OCH}_{3}\right), 100.1\left(\mathrm{C}_{8}\right)$, $105.2\left(\mathrm{C}_{5}\right), 112.4\left(\mathrm{C}_{4 \mathrm{a}}\right), 112.5\left(\mathrm{C}_{3}\right), 146.2\left(\mathrm{C}_{4}\right), 149.2\left(\mathrm{C}_{8 \mathrm{a}}\right)$, $152.3,159.1 \quad\left(\mathrm{C}_{6}, \mathrm{C}_{7}\right), 161.5(\mathrm{CO}) ; \mathrm{MS}(\mathrm{EI}) \mathrm{m} / \mathrm{z}$ (rel intensity): $220\left(\mathrm{M}^{+}, 100\right), 192$ (10), 149 (14), 1201 (10); HRMS (ESI ${ }^{+}$) Calcd. for $\mathrm{C}_{12} \mathrm{H}_{13} \mathrm{O}_{4}\left[\mathrm{MH}^{+}\right]:$221.0808; found, 221.0820 .

5,6,7-Trimethoxy-4-methyl-2H-chromen-2-one (2r). Coumarin $2 \mathrm{r}$ was prepared from $1 \mathbf{r}(674 \mathrm{mg}, 0.24 \mathrm{mmol}), 1$-fluoro2,4,6-trimethylpyridinium trifluoromethanesulfonate $(88.3 \mathrm{mg}$, $0.31 \mathrm{mmol}), \mathrm{Cu}(\mathrm{OAc})_{2}(4.4 \mathrm{mg}, 0.024 \mathrm{mmol}), p-\mathrm{TsOH}(48.8$ $\mathrm{mg}, 0.24 \mathrm{mmol})$, and $\mathrm{PdCl}_{2}\left(\mathrm{CH}_{3} \mathrm{CN}\right)_{2}(6.2 \mathrm{mg}, 0.024 \mathrm{mmol})$ in mesitylene $(1.0 \mathrm{~mL})$. The mixture was stirred at $70{ }^{\circ} \mathrm{C}$ for $72 \mathrm{~h}$, and after the work-up, flash column chromatography (silica gel, petroleum ether/EtOAc 8/2) afforded 2 r (13.0 mg, $18 \%)$ as an oil, whose data are coincidental with those reported: ${ }^{74} \mathrm{IR}$ (ATR): $1735 \mathrm{~cm}^{-1}(\mathrm{C}=\mathrm{O}) ;{ }^{1} \mathrm{H} \mathrm{NMR}\left(\mathrm{CDCl}_{3}\right.$, $300 \mathrm{MHz}): \delta 2.56\left(\mathrm{~d}, J=1.1 \mathrm{~Hz}, 3 \mathrm{H}, \mathrm{CH}_{3}\right), 3.85(\mathrm{~s}, 3 \mathrm{H}$, $\left.\mathrm{OCH}_{3}\right), 3.91\left(\mathrm{~s}, 3 \mathrm{H}, \mathrm{OCH}_{3}\right), 3.96\left(\mathrm{~s}, 3 \mathrm{H}, \mathrm{OCH}_{3}\right), 6.04(\mathrm{~d}, J=$ $\left.1.1 \mathrm{~Hz}, 1 \mathrm{H}, \mathrm{H}_{3}\right), 6.64\left(\mathrm{~s}, 1 \mathrm{H}, \mathrm{H}_{8}\right) ;{ }^{13} \mathrm{C}\left\{{ }^{1} \mathrm{H}\right\}$ NMR $\left(\mathrm{CDCl}_{3}\right.$, $75.5 \mathrm{MHz}): \delta 23.0\left(\mathrm{CH}_{3}\right), 56.2\left(\mathrm{OCH}_{3}\right), 61.0\left(\mathrm{OCH}_{3}\right), 61.3$ $\left(\mathrm{OCH}_{3}\right), 96.2\left(\mathrm{C}_{8}\right), 108.1\left(\mathrm{C}_{4 \mathrm{a}}\right), 113.0\left(\mathrm{C}_{3}\right), 139.2\left(\mathrm{C}_{6}\right), 151.3$ $\left(\mathrm{C}_{8 \mathrm{a}}\right), 151.6\left(\mathrm{C}_{4}\right), 153.6,156.3\left(\mathrm{C}_{5}, \mathrm{C}_{7}\right), 160.1(\mathrm{CO})$; MS (EI) $\mathrm{m} / z$ (rel intensity): $250\left(\mathrm{M}^{+}, 90\right), 235$ (100), 207 (75), 163 (39), 149 (14), 69 (17), 53 (14), 51 (13); HRMS (ESI $\left.{ }^{+}\right)$ Calcd. for $\mathrm{C}_{13} \mathrm{H}_{15} \mathrm{O}_{5}\left[\mathrm{MH}^{+}\right]$: 251.0914; found, 251.0939.

4-Methyl-6,7-methylenedioxy-2H-chromen-6-one (2s). Coumarin $2 \mathrm{~s}$ was prepared from $1 \mathrm{~s}(61.5 \mathrm{mg}, 0.30 \mathrm{mmol})$, 1-fluoro-2,4,6-trimethylpyridinium trifluoromethanesulfonate (101.1 mg, $0.35 \mathrm{mmol}), \mathrm{Cu}(\mathrm{OAc})_{2}(5.1 \mathrm{mg}, 0.015 \mathrm{mmol})$, p-TsOH (57.7 mg, $0.30 \mathrm{mmol})$, and $\mathrm{PdCl}_{2}\left(\mathrm{CH}_{3} \mathrm{CN}\right)_{2}(8.0 \mathrm{mg}$, $0.027 \mathrm{mmol})$ in mesitylene $(1.5 \mathrm{~mL})$. The mixture was stirred at $70{ }^{\circ} \mathrm{C}$ for $96 \mathrm{~h}$, and after the work-up, flash column chromatography (silica gel, petroleum ether/EtOAc 7/3) afforded $2 \mathrm{~s}(13.0 \mathrm{mg}, 20 \%)$ as a solid, whose data are coincidental with those reported: ${ }^{75} \mathrm{mp}\left(\mathrm{CH}_{2} \mathrm{Cl}_{2}\right): 120-122$ ${ }^{\circ} \mathrm{C}$; IR (ATR): $1735 \mathrm{~cm}^{-1}(\mathrm{C}=\mathrm{O})$; ${ }^{1} \mathrm{H} \mathrm{NMR}\left(\mathrm{CDCl}_{3}, 300\right.$ $\mathrm{MHz}): \delta 2.37\left(\mathrm{~s}, 3 \mathrm{H}, \mathrm{CH}_{3}\right), 6.07\left(\mathrm{~s}, 2 \mathrm{H}, \mathrm{OCH}_{2} \mathrm{O}\right), 6.17(\mathrm{~s}$, $\left.1 \mathrm{H}, \mathrm{H}_{3}\right), 6.83\left(\mathrm{~s}, 1 \mathrm{H}, \mathrm{H}_{5}\right), 6.96\left(\mathrm{~s}, 1 \mathrm{H}, \mathrm{H}_{8}\right) ;{ }^{13} \mathrm{C}\left\{{ }^{1} \mathrm{H}\right\} \mathrm{NMR}$ $\left(\mathrm{CDCl}_{3}, 75.5 \mathrm{MHz}\right): \delta 19.1\left(\mathrm{CH}_{3}\right), 98.4\left(\mathrm{OCH}_{2} \mathrm{O}\right), 102.1$ $\left(\mathrm{C}_{8}\right), 102.3\left(\mathrm{C}_{5}\right), 112.2\left(\mathrm{C}_{4 \mathrm{a}}\right), 113.8\left(\mathrm{C}_{3}\right), 144.9\left(\mathrm{C}_{4}\right), 150.6$ $\left(\mathrm{C}_{8 \mathrm{a}}\right), 150.9,152.4\left(\mathrm{C}_{6}, \mathrm{C}_{7}\right), 161.2(\mathrm{CO}) ; \mathrm{MS}(\mathrm{EI}) \mathrm{m} / z$ (rel intensity): $204\left(\mathrm{M}^{+}, 100\right), 176$ (72), 96 (15); HRMS $\left(\mathrm{ESI}^{+}\right)$ Calcd. for $\mathrm{C}_{11} \mathrm{H}_{9} \mathrm{O}_{4}\left[\mathrm{MH}^{+}\right]$: 205.0495; found, 205.0523 .

(Z)-3-(3,5-Difluorobenzylidene)-4,6-dimethoxybenzofuran-2(3H)-ne $(3 \mathrm{~g})$. Compound $3 \mathrm{~g}$ was obtained from $\mathbf{1 g}(123.8$ $\mathrm{mg}, 0.39 \mathrm{mmol})$, 1-fluoro-2,4,6-trimethylpyridinium trifluoromethanesulfonate $(135.5 \mathrm{mg}, 0.46 \mathrm{mmol}), \mathrm{Cu}(\mathrm{OAc})_{2}$ (3.6 $\mathrm{mg}, 0.019 \mathrm{mmol}), p-\mathrm{TsOH}(74.6 \mathrm{mg}, 0.39 \mathrm{mmol})$, and $\mathrm{PdCl}_{2}\left(\mathrm{CH}_{3} \mathrm{CN}\right)_{2}(5.1 \mathrm{mg}, 0.019 \mathrm{mmol})$ in HOAc $(1.5 \mathrm{~mL})$. The mixture was stirred at $70{ }^{\circ} \mathrm{C}$ for $24 \mathrm{~h}$, and after the workup, flash column chromatography (silica gel, hexane/EtOAc 8/ 2) afforded $2 \mathrm{~g}(9.8 \mathrm{mg}, 8 \%)$ and $3 \mathrm{~g}(18.3 \mathrm{mg}, 15 \%)$ as a solid: mp $\left(\mathrm{CH}_{2} \mathrm{Cl}_{2}\right): 202-204{ }^{\circ} \mathrm{C}$; IR (ATR): $1774 \mathrm{~cm}^{-1}(\mathrm{C}=\mathrm{O})$; ${ }^{1} \mathrm{H}$ NMR $\left(\mathrm{CDCl}_{3}, 300 \mathrm{MHz}\right): \delta 3.85\left(\mathrm{~s}, 3 \mathrm{H}, \mathrm{OCH}_{3}\right), 3.97(\mathrm{~s}$, $\left.3 \mathrm{H}, \mathrm{OCH}_{3}\right), 6.26\left(\mathrm{~d}, J=2.0 \mathrm{~Hz}, 1 \mathrm{H}, \mathrm{H}_{5}\right), 6.32(\mathrm{~d}, J=2.0 \mathrm{~Hz}$, $\left.1 \mathrm{H}, \mathrm{H}_{7}\right), 6.79-6.88\left(\mathrm{~m}, 1 \mathrm{H}, \mathrm{H}_{4^{\prime}}\right), 7.49-7.63\left(\mathrm{~m}, 1 \mathrm{H}, \mathrm{H}_{2^{\prime}}\right.$, $\left.\mathrm{H}_{6^{\prime}}\right), 7.80(\mathrm{~s}, 1 \mathrm{H}, \mathrm{C}=\mathrm{C} \underline{\mathrm{H}}-\mathrm{Ar}) ;{ }^{13} \mathrm{C}\left\{{ }^{1} \mathrm{H}\right\} \operatorname{NMR}\left(\mathrm{CDCl}_{3}, 75.5\right.$ $\mathrm{MHz}): \delta 55.8\left(\mathrm{OCH}_{3}\right), 55.9\left(\mathrm{OCH}_{3}\right), 89.3\left(\mathrm{C}_{7}\right), 94.3\left(\mathrm{C}_{5}\right)$, 104.7-105.8 (m, $\left.\mathrm{C}_{3 a}, \mathrm{C}_{4^{\prime}}\right), 113.5-113.9\left(\mathrm{~m}, \mathrm{C}_{2^{\prime}}, \mathrm{C}_{6^{\prime}}\right), 122.6$ $\left(\mathrm{C}_{3}\right), 136.4(\mathrm{C}=\underline{\mathrm{CH}}-\mathrm{Ar}), 136.9\left(\mathrm{t}, J=10.0 \mathrm{~Hz}, \mathrm{C}_{1^{\prime}}\right), 155.1$ $\left(\mathrm{C}_{7 \mathrm{a}}\right), 157.0\left(\mathrm{C}_{4}\right), 162.6\left(\mathrm{dd}, J=247.5,13.0 \mathrm{~Hz}, \mathrm{C}_{3^{\prime}}, \mathrm{C}_{5^{\prime}}\right)$, $163.1\left(\mathrm{C}_{6}\right), 165.8(\mathrm{CO})$; MS (EI) $\mathrm{m} / z$ (rel intensity) $319\left(\mathrm{M}^{+}\right.$ $+1,19), 318\left(\mathrm{M}^{+}, 100\right), 275$ (28), 188 (12), 175 (11), 69 
(10); HRMS (ESI ${ }^{+}$) Calcd. for $\mathrm{C}_{17} \mathrm{H}_{13} \mathrm{~F}_{2} \mathrm{O}_{4}\left[\mathrm{MH}^{+}\right]$: 319.0776; found, 319.0783 .

C3 Intermolecular C-H Alkenylation of Coumarins 2: General Procedure. Over a solution of coumarins 2 (1 $\mathrm{mmol})$ in 1,4-dioxane $(5 \mathrm{~mL}), \mathrm{AgOAc}(3 \mathrm{mmol}), \mathrm{PivOH}(10$ $\mathrm{mmol})$, and $\mathrm{Pd}(\mathrm{OAc})_{2}(0.1$ or $0.2 \mathrm{mmol})$ were added. The mixture was stirred vigorously for $5 \mathrm{~min}$, and then the corresponding acrylate ( 2 or $4 \mathrm{mmol}$ ) was added. The reaction was heated at $120^{\circ} \mathrm{C}$ for $24-48 \mathrm{~h}$, and then the reaction was allowed to cool down to room temperature. The mixture was filtered through diatomaceus earth and the filtrate was concentrated in vacuo. Flash column chromatography (silica gel, hexane/EtOAc) afforded coumarins 4.

Methyl (E)-3-(5,7-Dimethoxy-2-oxo-4-phenyl-2H-chromen-3-yl)acrylate (4a). Coumarin $4 a$ was prepared from $2 \mathrm{a}$ $(83.2 \mathrm{mg}, 0.29 \mathrm{mmol}), \mathrm{AgOAc}(0.15 \mathrm{~g}, 0.88 \mathrm{mmol}), \mathrm{PivOH}$ $(0.30 \mathrm{~g}, 2.95 \mathrm{mmol})$, methylacrylate $(0.06 \mathrm{~mL}, 0.59 \mathrm{mmol})$, and $\mathrm{Pd}(\mathrm{OAc})_{2}(6.6 \mathrm{mg}, 0.029 \mathrm{mmol})$. The reaction was heated at $120{ }^{\circ} \mathrm{C}$ for $24 \mathrm{~h}$, and after this, additional amounts of methyl acrylate $(0.06 \mathrm{~mL}, 0.59 \mathrm{mmol})$ and $\mathrm{Pd}(\mathrm{OAc})_{2}(6.6 \mathrm{mg}, 0.029$ mmol) were added, and it was heated again at $120^{\circ} \mathrm{C}$ for $24 \mathrm{~h}$. After the work-up, flash column chromatography (silica gel, hexane/EtOAc 7/3) afforded $4 \mathrm{a}(33.4 \mathrm{mg}, 31 \%)$ as a solid: $\mathrm{mp}$ $\left(\mathrm{CH}_{2} \mathrm{Cl}_{2}\right)$ 194-196 ${ }^{\circ} \mathrm{C}$; IR (ATR): $1710 \mathrm{~cm}^{-1}(\mathrm{C}=\mathrm{O}) ;{ }^{1} \mathrm{H}$ NMR $\left(\mathrm{CDCl}_{3}, 300 \mathrm{MHz}\right): \delta 3.29(\mathrm{~s}, 3 \mathrm{H}, \mathrm{CH}=\mathrm{CH}-$ $\left.\mathrm{COOCH}_{3}\right), 3.65\left(\mathrm{~s}, 3 \mathrm{H}, \mathrm{OCH}_{3}\right), 3.87\left(\mathrm{~s}, 3 \mathrm{H}, \mathrm{OCH}_{3}\right), 6.15$ $\left(\mathrm{d}, J=2,4 \mathrm{~Hz}, 1 \mathrm{H}, \mathrm{H}_{6}\right), 6.49\left(\mathrm{~d}, J=2,4 \mathrm{~Hz}, 1 \mathrm{H}, \mathrm{H}_{8}\right), 7.06-$ $7.15\left(\mathrm{~m}, 4 \mathrm{H}, \mathrm{H}_{2^{\prime}}, \mathrm{H}_{4^{\prime}}, \mathrm{H}_{6^{\prime}}, \mathrm{CH}=\mathrm{C} \underline{\mathrm{H}}-\mathrm{COOCH}_{3}\right), 7.34-7.47$ $\left(\mathrm{m}, 3 \mathrm{H}, \mathrm{H}_{3^{\prime}}, \mathrm{H}_{5^{\prime}}, \mathrm{CH}=\mathrm{CH}-\mathrm{COOCH}_{3}\right) ;{ }^{13} \mathrm{C}\left\{{ }^{1} \mathrm{H}\right\} \quad \mathrm{NMR}$ $\left(\mathrm{CDCl}_{3}, 75.5 \mathrm{MHz}\right): \delta 51.4\left(\mathrm{CH}=\mathrm{CH}-\mathrm{COOCH}_{3}\right), 55.7$ $\left(\mathrm{OCH}_{3}\right), 55.9\left(\mathrm{OCH}_{3}\right), 93.1\left(\mathrm{C}_{8}\right), 96.0\left(\mathrm{C}_{6}\right), 104.7\left(\mathrm{C}_{4 \mathrm{a}}\right)$, $115.7\left(\mathrm{C}_{3}\right), 121.9\left(\mathrm{CH}=\underline{\mathrm{CH}}-\mathrm{COOCH}_{3}\right), 127.1\left(\mathrm{C}_{2^{\prime}}, \mathrm{C}_{6^{\prime}}\right)$, $127.8\left(\mathrm{C}_{3^{\prime}}, \mathrm{C}_{5^{\prime}}\right), 127.9\left(\mathrm{C}_{4^{\prime}}\right), 137.2\left(\mathrm{CH}=\mathrm{CH}-\mathrm{COOCH}_{3}\right)$, $137.7\left(\mathrm{C}_{1^{\prime}}\right), 155.8\left(\mathrm{C}_{4}\right), 156.0\left(\mathrm{C}_{8 \mathrm{a}}\right), 159.1,164.4\left(\mathrm{C}_{5}, \mathrm{C}_{7}\right)$ 159.2 (CO), $168.4\left(\mathrm{COOCH}_{3}\right) ; \mathrm{MS}(\mathrm{CI}) \mathrm{m} / z$ (rel intensity) $368\left(\mathrm{MH}^{+}+1,19\right), 367\left(\mathrm{MH}^{+}, 100\right), 336(7), 335(41)$; HRMS (ESI ${ }^{+}$) Calcd. for $\mathrm{C}_{21} \mathrm{H}_{19} \mathrm{O}_{6}\left[\mathrm{MH}^{+}\right]$: 367.1176 ; found, 367.1180 .

tert-Butyl (E)-3-(5,7-Dimethoxy-2-oxo-4-phenyl-2H-chromen-3-yl)acrylate (4b). Coumarin $\mathbf{4 b}$ was prepared from $2 \mathrm{a}$ (82.4 mg, $0.29 \mathrm{mmol}), \mathrm{AgOAc}(0.15 \mathrm{~g}, 0.88 \mathrm{mmol}), \mathrm{PivOH}$ $(0.30 \mathrm{~g}, 2.92 \mathrm{mmol})$, tert-butyl acrylate $(85 \mu \mathrm{L}, 0.58 \mathrm{mmol})$, and $\mathrm{Pd}(\mathrm{OAc})_{2}(6.6 \mathrm{mg}, 0.029 \mathrm{mmol})$. The reaction was heated at $120{ }^{\circ} \mathrm{C}$ for $24 \mathrm{~h}$, and after the work-up, flash column chromatography (silica gel, $\left.\mathrm{CH}_{2} \mathrm{Cl}_{2}\right)$ afforded $4 \mathbf{b}(37.3 \mathrm{mg}$, $31 \%, 44 \%$ conversion) as a solid: $\mathrm{mp}\left(\mathrm{CH}_{2} \mathrm{Cl}_{2}\right) 148-150{ }^{\circ} \mathrm{C}$; IR (ATR): 1732 (COO), $1706(\mathrm{COO} t \mathrm{Bu}) \mathrm{cm}^{-1} ;{ }^{1} \mathrm{H}$ NMR $\left(\mathrm{CDCl}_{3}, 300 \mathrm{MHz}\right): \delta 1.41\left(\mathrm{~s}, 9 \mathrm{H}, \mathrm{C}\left(\mathrm{CH}_{3}\right)_{3}\right), 3.31(\mathrm{~s}, 3 \mathrm{H}$, $\left.\mathrm{OCH}_{3}\right), 3.89\left(\mathrm{OCH}_{3}\right), 6.17\left(\mathrm{~d}, J=2.4 \mathrm{~Hz}, 1 \mathrm{H}, \mathrm{H}_{6}\right), 6.52(\mathrm{~d}, J$ $\left.=2.4 \mathrm{~Hz}, 1 \mathrm{H}, \mathrm{H}_{8}\right), 7.02-7.15\left(\mathrm{~m}, 4 \mathrm{H}, \mathrm{H}_{2^{\prime}}, \mathrm{H}_{4^{\prime}}, \mathrm{H}_{6^{\prime}}, \mathrm{CH}=\right.$ $\mathrm{C} \underline{\mathrm{H}}-\mathrm{COO} t \mathrm{Bu}), 7.35-7.47\left(\mathrm{~m}, 3 \mathrm{H}, \mathrm{H}_{3^{\prime}}, \mathrm{H}_{5^{\prime}}, \mathrm{CH}=\mathrm{CH}-\right.$ COOtBu $) ;{ }^{13} \mathrm{C}\left\{{ }^{1} \mathrm{H}\right\} \quad \mathrm{NMR}\left(\mathrm{CDCl}_{3}, 75.5 \mathrm{MHz}\right): \delta 28.1$ $\left(\mathrm{C}\left(\underline{\mathrm{CH}}_{3}\right)_{3}\right), 55.7\left(\mathrm{OCH}_{3}\right), 55.9\left(\mathrm{OCH}_{3}\right), 79.9\left(\underline{\mathrm{C}}\left(\mathrm{CH}_{3}\right)_{3}\right)$, 93.1 $\left(\mathrm{C}_{8}\right)$, $96.0\left(\mathrm{C}_{6}\right), 104.8\left(\mathrm{C}_{4 \mathrm{a}}\right), 115.9\left(\mathrm{C}_{3}\right), 124.4(\mathrm{CH}=$ $\underline{\mathrm{C}} \mathrm{H}-\mathrm{COO} t \mathrm{Bu}), 127.1\left(\mathrm{C}_{3^{\prime}}, \mathrm{C}_{5^{\prime}}\right), 127.7\left(\mathrm{C}_{4^{\prime}}\right), 127.9\left(\mathrm{C}_{2^{\prime}}, \mathrm{C}_{6^{\prime}}\right)$, $135.9(\underline{\mathrm{CH}}=\mathrm{CH}-\mathrm{COO} t \mathrm{Bu}), 137.8\left(\mathrm{C}_{1^{\prime}}\right), 155.4,155.9\left(\mathrm{C}_{4}\right.$, $\left.\mathrm{C}_{8 \mathrm{a}}\right), 159.1,159.2\left(\mathrm{C}_{5}, \mathrm{C}_{7}\right), 164.2(\mathrm{CO}), 166.9(\mathrm{COO} t \mathrm{Bu})$; MS $\left(\mathrm{ESI}^{+}\right) \mathrm{m} / z$ (rel intensity) $410\left(\mathrm{MH}^{+}+1,9\right), 409\left(\mathrm{MH}^{+}\right.$, 45), 354 (16), 353 (100); HRMS (ESI ${ }^{+}$Calcd. for $\mathrm{C}_{25} \mathrm{H}_{25} \mathrm{O}_{6}$ $\left[\mathrm{MH}^{+}\right]$: 409.1651; found, 409.1653.

Methyl (E)-3-\{4-[4-(tert-Butyl)phenyl]-5,7-dimethoxy-2oxo-2H-chromen-3-ylacrylate (4c). Coumarin $4 \mathrm{c}$ was prepared from $2 \mathrm{~d}(85.0 \mathrm{mg}, 0.25 \mathrm{mmol}), \mathrm{AgOAc}(0.13 \mathrm{~g}, 0.75$ $\mathrm{mmol})$, PivOH (0.26 g, $2.51 \mathrm{mmol})$, methyl acrylate $(45 \mu \mathrm{L}$, $0.50 \mathrm{mmol})$, and $\mathrm{Pd}(\mathrm{OAc})_{2}(5.6 \mathrm{mg}, 0.025 \mathrm{mmol})$. The reaction was heated at $120{ }^{\circ} \mathrm{C}$ for $24 \mathrm{~h}$, and after this, additional amounts of methyl acrylate $(45 \mu \mathrm{L}, 0.50 \mathrm{mmol})$ and $\mathrm{Pd}(\mathrm{OAc})_{2}(5.6 \mathrm{mg}, 0.025 \mathrm{mmol})$ were added, and it was heated again at $120{ }^{\circ} \mathrm{C}$ for $24 \mathrm{~h}$. After the work-up, flash column chromatography (silica gel, petroleum ether/EtOAc 8/ 2) afforded $4 \mathrm{c}$ (55.4 $\mathrm{mg}, 52 \%, 63 \%$ conversion) as a solid: $\mathrm{mp}$ $\left(\mathrm{CH}_{2} \mathrm{Cl}_{2}\right): 159-162{ }^{\circ} \mathrm{C}$; IR (ATR): $1726 \mathrm{~cm}^{-1}(\mathrm{C}=\mathrm{O}) ;{ }^{1} \mathrm{H}$ NMR $\left(\mathrm{CDCl}_{3}, 300 \mathrm{MHz}\right): \delta 1.40\left(\mathrm{~s}, 9 \mathrm{H}, \mathrm{C}\left(\mathrm{CH}_{3}\right)_{3}\right), 3.28(\mathrm{~s}$, $\left.3 \mathrm{H}, \mathrm{COOCH}_{3}\right), 3.67\left(\mathrm{~s}, 3 \mathrm{H}, \mathrm{OCH}_{3}\right), 3.88\left(\mathrm{~s}, 3 \mathrm{H}, \mathrm{OCH}_{3}\right)$, $6.16\left(\mathrm{~d}, J=2.4 \mathrm{~Hz}, 1 \mathrm{H}, \mathrm{H}_{6}\right), 6.50\left(\mathrm{~d}, J=2.4 \mathrm{~Hz}, 1 \mathrm{H}, \mathrm{H}_{8}\right)$, $7.04-7.06\left(\mathrm{~m}, 2 \mathrm{H}, \mathrm{H}_{3^{\prime}}, \mathrm{H}_{5^{\prime}}\right), 7.16(\mathrm{~d}, J=15.8 \mathrm{~Hz}, 1 \mathrm{H}, \mathrm{CH}=$ $\left.\mathrm{C} \mathrm{H}-\mathrm{COOCH}_{3}\right), 7.24(\mathrm{~d}, J=15.8 \mathrm{~Hz}, 1 \mathrm{H}, \mathrm{CH}=\mathrm{CH}-$ $\left.\mathrm{COOCH}_{3}\right)$, 7.44-7.47 (m, 2H, $\left.\mathrm{H}_{2}, \mathrm{H}_{6^{\prime}}\right) ;{ }^{13} \mathrm{C}\left\{{ }^{1} \mathrm{H}\right\}$ NMR $\left(\mathrm{CDCl}_{3}, 75.5 \mathrm{MHz}\right): \delta 31.3\left(\mathrm{C}\left(\mathrm{CH}_{3}\right)_{3}\right), 34.7\left(\underline{\mathrm{C}}\left(\mathrm{CH}_{3}\right)_{3}\right), 51.4$ $\left(\mathrm{COOCH}_{3}\right), 55.8\left(\mathrm{OCH}_{3}\right), 55.8\left(\mathrm{OCH}_{3}\right), 93.1\left(\mathrm{C}_{8}\right), 96.1$ $\left(\mathrm{C}_{6}\right), 105.0\left(\mathrm{C}_{\mathrm{Aa}_{\mathrm{a}}}\right), 115.7\left(\mathrm{C}_{3}\right), 121.8\left(\mathrm{CH}=\mathrm{CH}-\mathrm{COOCH}_{3}\right)$, $124.7\left(\mathrm{C}_{3^{\prime}}, \mathrm{C}_{5^{\prime}}\right), 126.9\left(\mathrm{C}_{2^{\prime}}, \mathrm{C}_{6^{\prime}}\right), 134.6\left(\mathrm{C}_{1^{\prime}}\right), 137.4(\mathrm{CH}=$ $\left.\mathrm{CH}-\mathrm{COOCH}_{3}\right), 150.8\left(\mathrm{C}_{4^{\prime}}\right), 156.0\left(\mathrm{C}_{4}, \mathrm{C}_{8 \mathrm{a}}\right), 156.3,159.2$ $\left(\mathrm{C}_{5}, \mathrm{C}_{7}\right), 164.3(\mathrm{CO}), 168.3\left(\mathrm{COOCH}_{3}\right) ; \mathrm{MS}\left(\mathrm{ESI}^{+}\right) \mathrm{m} / z$ (rel intensity) $424\left(\mathrm{MH}^{+}+1,24\right), 423\left(\mathrm{MH}^{+}, 100\right), 391(20), 318$ (11); HRMS (ESI ${ }^{+}$) Calcd. for $\mathrm{C}_{25} \mathrm{H}_{27} \mathrm{O}_{6}\left[\mathrm{MH}^{+}\right]$: 423.1808; found, 423.1801 .

Methyl (E)-3-[4-(3,5-Dimethoxyphenyl)-5,7-dimethoxy-2oxo-2H-chromen-3-yl]acrylate (4d). Coumarin $4 \mathrm{~d}$ was prepared from $2 \mathrm{e}(69.2 \mathrm{mg}, 0.20 \mathrm{mmol}), \mathrm{AgOAc}(0.10 \mathrm{~g}$, $0.61 \mathrm{mmol}), \mathrm{PivOH}(0.21 \mathrm{~g}, 2.02 \mathrm{mmol})$, methyl acrylate (36 $\mu \mathrm{L}, 0.40 \mathrm{mmol})$, and $\mathrm{Pd}(\mathrm{OAc})_{2}(4.5 \mathrm{mg}, 0.020 \mathrm{mmol})$. The reaction was heated at $120{ }^{\circ} \mathrm{C}$ for $24 \mathrm{~h}$, and after this, additional amounts of methyl acrylate $(36 \mu \mathrm{L}, 0.40 \mathrm{mmol})$ and $\mathrm{Pd}(\mathrm{OAc})_{2}(4.5 \mathrm{mg}, 0.020 \mathrm{mmol})$ were added, and it was heated again at $120{ }^{\circ} \mathrm{C}$ for $24 \mathrm{~h}$. After the work-up, flash column chromatography (silica gel, petroleum ether/EtOAc 6/ 4) afforded $\mathbf{4 d}$ (24.2 $\mathrm{mg}, 28 \%, 57 \%$ conversion) as a solid: $\mathrm{mp}$ $\left(\mathrm{CH}_{2} \mathrm{Cl}_{2}\right) 207-209{ }^{\circ} \mathrm{C}$; IR (ATR): $1722 \mathrm{~cm}^{-1}(\mathrm{C}=\mathrm{O}) ;{ }^{1} \mathrm{H}$ NMR $\left(\mathrm{CDCl}_{3}, 300 \mathrm{MHz}\right): \delta 3.40\left(\mathrm{~s}, 3 \mathrm{H}, \mathrm{COOCH}_{3}\right), 3.69(\mathrm{~s}$, $\left.3 \mathrm{H}, \mathrm{OCH}_{3}\right), 3.81\left(\mathrm{~s}, 6 \mathrm{H}, 2 \times \mathrm{OCH}_{3}\right), 3.88\left(\mathrm{~s}, 3 \mathrm{H}, \mathrm{OCH}_{3}\right)$, $6.19\left(\mathrm{~d}, J=2.4 \mathrm{~Hz}, 1 \mathrm{H}, \mathrm{H}_{6}\right), 6.28-6.29\left(\mathrm{~m}, 2 \mathrm{H}, \mathrm{H}_{2}, \mathrm{H}_{6^{\prime}}\right)$, 6.49-6.51 (m, 2H, $\left.\mathrm{H}_{8}, \mathrm{H}_{4^{\prime}}\right), 7.15-7.27(\mathrm{~m}, 2 \mathrm{H}, \mathrm{CH}=\mathrm{C} \underline{\mathrm{H}}-$ $\left.\mathrm{COOCH}_{3}\right) ;{ }^{13} \mathrm{C}\left\{{ }^{1} \mathrm{H}\right\}$ NMR $\left(\mathrm{CDCl}_{3}, 75.5 \mathrm{MHz}\right): \delta 51.5$ $\left(\mathrm{COOCH}_{3}\right), 55.5\left(2 \times \mathrm{OCH}_{3}\right), 55.9\left(\mathrm{OCH}_{3}\right), 56.0\left(\mathrm{OCH}_{3}\right)$, 93.1 $\left(\mathrm{C}_{8}\right)$, $96.1\left(\mathrm{C}_{6}\right), 99.8\left(\mathrm{C}_{4^{\prime}}\right), 104.6\left(\mathrm{C}_{4 \mathrm{a}}\right), 105.5\left(\mathrm{C}_{2^{\prime}}, \mathrm{C}_{6^{\prime}}\right)$, $115.5\left(\mathrm{C}_{3}\right), 122.0\left(\mathrm{CH}=\underline{\mathrm{C}} \mathrm{H}-\mathrm{COOCH}_{3}\right), 137.2(\underline{\mathrm{CH}}=\mathrm{CH}-$ $\left.\mathrm{COOCH}_{3}\right), 139.5\left(\mathrm{C}_{1^{\prime}}\right), 155.5\left(\mathrm{C}_{4}\right), 156.0\left(\mathrm{C}_{8 \mathrm{a}}\right), 159.2,160.6$ $\left(\mathrm{C}_{5}, \mathrm{C}_{7}\right), 164.4(\mathrm{CO}), 168.2\left(\mathrm{COOCH}_{3}\right) ; \mathrm{MS}\left(\mathrm{ESI}^{+}\right) \mathrm{m} / z$ (rel intensity) $428\left(\mathrm{MH}^{+}+1,19\right), 427\left(\mathrm{MH}^{+}, 100\right), 396(10), 395$ (56), 353 (24); HRMS (ESI ${ }^{+}$) Calcd. for $\mathrm{C}_{23} \mathrm{H}_{23} \mathrm{O}_{8}\left[\mathrm{MH}^{+}\right]$: 427.1393; found, 427.1393 .

Methyl (E)-3-(5,7-Dimethoxy-4-methyl-2-oxo-2H-chromen-3-yl)acrylate (4e). Coumarin $4 \mathrm{e}$ was prepared from $2 \mathrm{i}$ (81.3 mg, $0.37 \mathrm{mmol}), \mathrm{AgOAc}(0.19 \mathrm{~g}, 1.11 \mathrm{mmol}), \mathrm{PivOH}$ $(0.38 \mathrm{~g}, 3.69 \mathrm{mmol})$, methyl acrylate $(0.07 \mathrm{~mL}, 0.74 \mathrm{mmol})$, and $\mathrm{Pd}(\mathrm{OAc})_{2}(8.5 \mathrm{mg}, 0.04 \mathrm{mmol})$. The reaction was heated at $120{ }^{\circ} \mathrm{C}$ for $48 \mathrm{~h}$, and after the work-up, flash column chromatography (silica gel, hexane/EtOAc 6/4) afforded $4 \mathrm{e}$ (50.7 mg, 45\%) as a solid: $\mathrm{mp}\left(\mathrm{CH}_{2} \mathrm{Cl}_{2}\right) 199-201{ }^{\circ} \mathrm{C}$; IR (ATR): $1713 \mathrm{~cm}^{-1}\left(\mathrm{COO}, \mathrm{COOCH}_{3}\right) ;{ }^{1} \mathrm{H} \mathrm{NMR}\left(\mathrm{CDCl}_{3}, 300\right.$ $\mathrm{MHz}): \delta 2.72\left(\mathrm{~s}, 3 \mathrm{H}, \mathrm{CH}_{3}\right), 3.79\left(\mathrm{~s}, 3 \mathrm{H}, \mathrm{COOCH}_{3}\right), 3.85(\mathrm{~s}$, $\left.3 \mathrm{H}, \mathrm{OCH}_{3}\right), 3.88\left(\mathrm{~s}, 3 \mathrm{H}, \mathrm{OCH}_{3}\right), 6.30\left(\mathrm{~d}, J=2,4 \mathrm{~Hz}, 1 \mathrm{H}, \mathrm{H}_{6}\right)$, $6.40\left(\mathrm{~d}, J=2,4 \mathrm{~Hz}, 1 \mathrm{H}, \mathrm{H}_{8}\right), 7.07(\mathrm{~d}, J=15,7 \mathrm{~Hz}, 1 \mathrm{H}, \mathrm{CH}=$ $\left.\mathrm{CH}-\mathrm{COOCH}_{3}\right), 7.82(\mathrm{~d}, J=15,7 \mathrm{~Hz}, 1 \mathrm{H}, \mathrm{CH}=\mathrm{CH}-$ $\left.\mathrm{COOCH}_{3}\right) ;{ }^{13} \mathrm{C}\left\{{ }^{1} \mathrm{H}\right\}$ NMR $\left(\mathrm{CDCl}_{3}, 75.5 \mathrm{MHz}\right): \delta 19.7$ 
$\left(\mathrm{CH}_{3}\right), 51.7\left(\mathrm{CH}=\mathrm{CH}-\mathrm{COOCH}_{3}\right), 55.8\left(\mathrm{OCH}_{3}\right), 55.9$ $\left(\mathrm{OCH}_{3}\right), 93.1\left(\mathrm{C}_{8}\right), 96.0\left(\mathrm{C}_{6}\right), 106.5\left(\mathrm{C}_{4 \mathrm{a}}\right), 115.5\left(\mathrm{C}_{3}\right)$, $123.1\left(\mathrm{CH}=\underline{\mathrm{CH}}-\mathrm{COOCH}_{3}\right), 136.3\left(\underline{\mathrm{CH}}=\mathrm{CH}-\mathrm{COOCH}_{3}\right)$, $154.5\left(\mathrm{C}_{4}\right), 155.8\left(\mathrm{C}_{8 \mathrm{a}}\right), 159.0,163.5\left(\mathrm{C}_{5}, \mathrm{C}_{7}\right), 159.8(\mathrm{CO})$, $168.4\left(\mathrm{COOCH}_{3}\right) ; \mathrm{MS}(\mathrm{CI}) \mathrm{m} / z$ (rel intensity) $306\left(\mathrm{MH}^{+}+\right.$ 1, 14), $305\left(\mathrm{MH}^{+}, 100\right), 274$ (10), 273 (85); HRMS (CI) Calcd. for $\mathrm{C}_{16} \mathrm{H}_{17} \mathrm{O}_{6}\left[\mathrm{MH}^{+}\right]$: 305.1020; found, 305.1021.

tert-Butyl (E)-3-(5,7-Dimethoxy-4-methyl-2-oxo-2H-chromen-3-yl)acrylate (4f). Coumarin $4 \mathbf{f}$ was prepared from $2 \mathbf{i}(60$ $\mathrm{mg}, 0.27 \mathrm{mmol}), \mathrm{AgOAc}(0.14 \mathrm{~g}, 0.82 \mathrm{mmol})$, PivOH (0.28 g, $2.72 \mathrm{mmol})$, tert-butyl acrylate $(0.08 \mathrm{~mL}, 0.54 \mathrm{mmol})$, and $\mathrm{Pd}(\mathrm{OAc})_{2}(6.2 \mathrm{mg}, 0.03 \mathrm{mmol})$. The reaction was heated at $120{ }^{\circ} \mathrm{C}$ for $24 \mathrm{~h}$, and after the work-up, flash column chromatography (silica gel, hexane/EtOAc 7/3) afforded 4f (49.8 mg, 53\%) as a solid: $\mathrm{mp}\left(\mathrm{CH}_{2} \mathrm{Cl}_{2}\right): 159-161{ }^{\circ} \mathrm{C}$; IR (ATR): 1716 (COO), 1695 (COOtBu) cm ${ }^{-1} ;{ }^{1} \mathrm{H}$ NMR $\left(\mathrm{CDCl}_{3}, 300 \mathrm{MHz}\right): \delta 1.51\left(\mathrm{~s}, 9 \mathrm{H}, \mathrm{C}\left(\mathrm{CH}_{3}\right)_{3}\right), 2.69(\mathrm{~s}, 3 \mathrm{H}$, $\left.\mathrm{CH}_{3}\right), 3.83\left(\mathrm{~s}, 3 \mathrm{H}, \mathrm{OCH}_{3}\right), 3.87\left(\mathrm{~s}, 3 \mathrm{H}, \mathrm{OCH}_{3}\right), 6.28(\mathrm{~d}, J=$ $\left.2.4 \mathrm{~Hz}, 1 \mathrm{H}, \mathrm{H}_{6}\right), 6.38\left(\mathrm{~d}, J=2.4 \mathrm{~Hz}, 1 \mathrm{H}, \mathrm{H}_{8}\right), 6.97$ (d, $J=15.7$ $\mathrm{Hz}, 1 \mathrm{H}, \mathrm{CH}=\mathrm{CH}-\mathrm{COO} t \mathrm{Bu}), 7.71(\mathrm{~d}, J=15.7 \mathrm{~Hz}, 1 \mathrm{H}$, $\mathrm{CH}=\mathrm{CH}-\mathrm{COO} t \mathrm{Bu}) ;{ }^{13} \mathrm{C}\left\{{ }^{1} \mathrm{H}\right\} \operatorname{NMR}\left(\mathrm{CDCl}_{3}, 75.5 \mathrm{MHz}\right): \delta$ $19.6\left(\mathrm{CH}_{3}\right), 28.2\left(\mathrm{C}\left(\underline{\mathrm{CH}}_{3}\right)_{3}\right), 55.7\left(\mathrm{OCH}_{3}\right), 55.9\left(\mathrm{OCH}_{3}\right)$, $80.3\left(\underline{\mathrm{C}}\left(\mathrm{CH}_{3}\right)_{3}\right), 93.1\left(\mathrm{C}_{8}\right), 95.9\left(\mathrm{C}_{6}\right), 105.5\left(\mathrm{C}_{4 \mathrm{a}}\right), 115.6$ $\left(\mathrm{C}_{3}\right), 125.4(\mathrm{CH}=\underline{\mathrm{C}} \mathrm{H}-\mathrm{COO} t \mathrm{Bu}), 135.0(\underline{\mathrm{CH}}=\mathrm{CH}-\mathrm{COO} t-$ $\mathrm{Bu}), 154.0\left(\mathrm{C}_{8 \mathrm{a}}\right), 155.7\left(\mathrm{C}_{4}\right), 159.1,159.7\left(\mathrm{C}_{5}, \mathrm{C}_{7}\right), 163.4$ (COO), 167.3 (COOtBu); MS (CI) $\mathrm{m} / z$ (rel intensity) 347.1 $\left(\mathrm{MH}^{+}, 6\right), 346.1$ (20), 292.1 (10), 291 (52), 290.1 (100), 274.1 (10), 273.1 (63), 262.1 (12), 245.1 (72); HRMS (CI) Calcd. for $\mathrm{C}_{19} \mathrm{H}_{23} \mathrm{O}_{6}\left[\mathrm{MH}^{+}\right]$: 347.1489; found, 347.1507.

Methyl (E)-3-(5,7-Dimethoxy-4-methyl-2-oxo-2H-chromen-3-yl)but-2-enoate (4g). Coumarin $\mathbf{4 g}$ was prepared from $2 \mathrm{i}(52.1 \mathrm{mg}, 0.24 \mathrm{mmol}), \mathrm{AgOAc}(0.12 \mathrm{~g}, 0.71 \mathrm{mmol})$, PivOH (0.24 g, $2.37 \mathrm{mmol})$, methyl crotonate $(0.05 \mathrm{~mL}, 0.47$ $\mathrm{mmol})$, and $\mathrm{Pd}(\mathrm{OAc})_{2}(5.4 \mathrm{mg}, 0.024 \mathrm{mmol})$. The reaction was heated at $120^{\circ} \mathrm{C}$ for $48 \mathrm{~h}$. After the work-up, flash column chromatography (silica gel, hexane/EtOAc 7/3) afforded $\mathbf{4 g}$ (33.5 mg, 44\%) as a solid: $\mathrm{mp}\left(\mathrm{CH}_{2} \mathrm{Cl}_{2}\right): 153-155{ }^{\circ} \mathrm{C}$; IR (ATR): $1706 \mathrm{~cm}^{-1}\left(\mathrm{COO}, \mathrm{COOCH}_{3}\right) ;{ }^{1} \mathrm{H} \mathrm{NMR}\left(\mathrm{CDCl}_{3}, 300\right.$ $\mathrm{MHz}): \delta 2.39\left(\mathrm{~d}, J=1.3 \mathrm{~Hz}, 3 \mathrm{H}, \mathrm{C}\left(\mathrm{CH}_{3}\right)=\mathrm{CH}\left(\mathrm{COOCH}_{3}\right)\right)$, $2.48\left(\mathrm{~s}, 3 \mathrm{H}, \mathrm{C}-\mathrm{CH}_{3}\right), 3.73\left(\mathrm{~s}, 3 \mathrm{H}, \mathrm{C}\left(\mathrm{CH}_{3}\right)=\mathrm{CH}-\right.$ $\left.\left(\mathrm{COOCH}_{3}\right)\right), 3.85\left(\mathrm{~s}, 3 \mathrm{H}, \mathrm{OCH}_{3}\right), 3.86\left(\mathrm{~s}, 3 \mathrm{H}, \mathrm{OCH}_{3}\right)$, $5.76-5.79\left(\mathrm{~m}, 1 \mathrm{H}, \mathrm{C}\left(\mathrm{CH}_{3}\right)=\mathrm{CH}\left(\mathrm{COOCH}_{3}\right)\right), 6.31(\mathrm{~d}, J=$ $\left.2,4 \mathrm{~Hz}, 1 \mathrm{H}, \mathrm{H}_{6}\right), 6.43\left(\mathrm{~d}, J=2,4 \mathrm{~Hz}, 1 \mathrm{H}, \mathrm{H}_{8}\right) ;{ }^{13} \mathrm{C}\left\{{ }^{1} \mathrm{H}\right\} \mathrm{NMR}$ $\left(\mathrm{CDCl}_{3}, 75.5 \mathrm{MHz}\right): \delta 19.4\left(\mathrm{C}\left(\mathrm{CH}_{3}\right)=\mathrm{CH}\left(\mathrm{COOCH}_{3}\right)\right)$, $20.7\left(\mathrm{C}-\mathrm{CH}_{3}\right), 51.1 \quad\left(\mathrm{C}\left(\mathrm{CH}_{3}\right)=\mathrm{CH}\left(\mathrm{COO}^{-} \mathrm{H}_{3}\right)\right), 55.7$ $\left(\mathrm{OCH}_{3}\right), 55.8\left(\mathrm{OCH}_{3}\right), 93.2\left(\mathrm{C}_{8}\right), 95.8\left(\mathrm{C}_{6}\right), 104.8\left(\mathrm{C}_{4 \mathrm{a}}\right)$, $111.4\left(\mathrm{C}_{3}\right), 121.6\left(\mathrm{C}\left(\mathrm{CH}_{3}\right)=\underline{\mathrm{CH}}\left(\mathrm{COOCH}_{3}\right)\right), 148.8(\underline{\mathrm{C}}-$ $\left.\left(\mathrm{CH}_{3}\right)=\mathrm{CH}\left(\mathrm{COOCH}_{3}\right)\right)$, $152.6\left(\mathrm{C}_{4}\right), 155.8\left(\mathrm{C}_{8 \mathrm{a}}\right), 159.4$, $159.5\left(\mathrm{C}_{5}, \mathrm{C}_{7}\right), 162.6(\mathrm{CO}), 166.5 \quad\left(\mathrm{C}\left(\mathrm{CH}_{3}\right)=\mathrm{CH}-\right.$ $\left.\left(\mathrm{COOCH}_{3}\right)\right)$; MS $\left(\mathrm{ESI}^{+}\right) \mathrm{m} / z$ (rel intensity) $319\left(\mathrm{MH}^{+}\right.$, 100), 288 (17), 287 (71); HRMS (ESI ${ }^{+}$) Calcd. for $\mathrm{C}_{17} \mathrm{H}_{19} \mathrm{O}_{6}$ $\left[\mathrm{MH}^{+}\right]$: 319.1176, found, 319.1171 .

\section{ASSOCIATED CONTENT}

\section{SI Supporting Information}

The Supporting Information is available free of charge at https://pubs.acs.org/doi/10.1021/acsomega.1c03469.

Preparation procedures for the substrates 1, additional substrates tested in the cyclization, additional essays for the intermolecular alkenylation reaction of $2 \mathbf{i}$, copies of the ${ }^{1} \mathrm{H}$ and ${ }^{13} \mathrm{C}$ NMR spectra of compounds 2-4, and the fluorescence spectra for selected compounds $\mathbf{2}$ and $\mathbf{4}$ (PDF)

\section{AUTHOR INFORMATION}

\section{Corresponding Authors}

Nuria Sotomayor - Departamento de Química Orgánica e Inorgánica, Facultad de Ciencia y Tecnología, Universidad del País Vasco/Euskal Herriko Unibertsitatea UPV/EHU, Bilbao 48080, Spain; 다이.org/0000-0003-3079-6380; Email: nuria.sotomayor@ehu.es

Esther Lete - Departamento de Química Orgánica e Inorgánica, Facultad de Ciencia y Tecnología, Universidad del País Vasco/Euskal Herriko Unibertsitatea UPV/EHU, Bilbao 48080, Spain; ○ orcid.org/0000-0001-8624-6842; Email: esther.lete@ehu.es

\section{Authors}

Verónica Ortiz-de-Elguea - Departamento de Química Orgánica e Inorgánica, Facultad de Ciencia y Tecnología, Universidad del País Vasco/Euskal Herriko Unibertsitatea UPV/EHU, Bilbao 48080, Spain

Asier Carral-Menoyo - Departamento de Química Orgánica e Inorgánica, Facultad de Ciencia y Tecnología, Universidad del País Vasco/Euskal Herriko Unibertsitatea UPV/EHU, Bilbao 48080, Spain

Lorena Simón-Vidal - Departamento de Química Orgánica e Inorgánica, Facultad de Ciencia y Tecnología, Universidad del País Vasco/Euskal Herriko Unibertsitatea UPV/EHU, Bilbao 48080, Spain

Mikel Martinez-Nunes - Departamento de Química Orgánica e Inorgánica, Facultad de Ciencia y Tecnología, Universidad del País Vasco/Euskal Herriko Unibertsitatea UPV/EHU, Bilbao 48080, Spain

Iratxe Barbolla - Departamento de Química Orgánica e Inorgánica, Facultad de Ciencia y Tecnología, Universidad del País Vasco/Euskal Herriko Unibertsitatea UPV/EHU, Bilbao 48080, Spain; Instituto Biofisika (UPV/EHU-CSIC), Leioa 48940, Spain

Marta G. Lete - CIC bioGUNE, Derio 48170, Spain; (1) orcid.org/0000-0002-9654-7881

Complete contact information is available at: https://pubs.acs.org/10.1021/acsomega.1c03469

\section{Notes}

The authors declare no competing financial interest.

\section{ACKNOWLEDGMENTS}

Ministerio de Economía y Competitividad (FEDER CTQ2016-74881-P), Ministerio de Ciencia e Innovación (PID2019-104148GB-I00), and Gobierno Vasco (IT104516) are gratefully acknowledged for their financial support. A.C.-M. wishes to thank Gobierno Vasco for a grant. Technical and human support provided by Servicios Generales de Investigación SGIker (UPV/EHU, MINECO, GV/EJ, ERDF and ESF) is also acknowledged.

\section{REFERENCES}

(1) Estévez-Braun, A.; González, A. G. Coumarins. Nat. Prod. Rep. 1997, 14, 465-475.

(2) Vazquez-Rodriguez, S.; Matos, M.; Borges, F.; Uriarte, E.; Santana, L. Bioactive Coumarins from Marine Sources: Origin, Structural Features and Pharmacological Properties. Curr. Top. Med. Chem. 2015, 15, 1755-1766. 
(3) Xu, J.; Kjer, J.; Sendker, J.; Wray, V.; Guan, H.; Edrada, R.; Müller, W. E. G.; Bayer, M.; Lin, W.; Wu, J.; Proksch, P. Cytosporones, coumarins, and an alkaloid from the endophytic fungus Pestalotiopsis sp. isolated from the Chinese mangrove plant Rhizophora mucronata. Bioorg. Med. Chem. 2009, 17, 7362-7367.

(4) Rateb, M. E.; Ebel, R. Secondary metabolites of fungi from marine habitats. Nat. Prod. Rep. 2011, 28, 290-344.

(5) Fan, H.; Peng, J.; Hamann, M. T.; Hu, J.-F. Lamellarins and Related Pyrrole-Derived Alkaloids from Marine Organisms. Chem. Rev. 2008, 108, 264-287.

(6) Fukuda, T.; Ishibashi, F.; Iwao, M. Lamellarin alkaloids: Isolation, synthesis, and biological activity. In The Alkaloids: Chemistry and Biology; Vol. 83, Knolker, H. J. Ed.; Elsevier: London, 2020; pp. $1-112$.

(7) Garg, S. S.; Gupta, J.; Sharma, S.; Sahu, D. An insight into the therapeutic applications of coumarin compounds and their mechanisms of action. Eur. J. Pharm. Sci. 2020, 152, 105424.

(8) Nagy, N.; Kuipers, H. F.; Frymoyer, A. R.; Ishak, H. D.; Bollyky, J. B.; Wight, T. N.; Bollyky, P. L. 4-Methylumbelliferone treatment and hyaluronan inhibition as a therapeutic strategy in inflammation, autoimmunity, and cancer. Front. Immunol. 2015, 6, 123.

(9) Kumagai, M.; Watanabe, A.; Yoshida, I.; Mishima, T.; Nakamura, M.; Nishikawa, K.; Morimoto, Y. Evaluation of Aculeatin and Toddaculin Isolated from Toddalia asiatica as Anti-inflammatory Agents in LPS-Stimulated RAW264 Macrophages. Biol. Pharm. Bull. 2018, 41, 132-137.

(10) McKee, T. C.; Fuller, R. W.; Covington, C. D.; Cardellina, J. H.; Gulakowski, R. J.; Krepps, B. L.; McMahon, J. B.; Boyd, M. R. New Pyranocoumarins Isolated from Calophyllum lanigerum and Calophyllum teysmannii. J. Nat. Prod. 1996, 59, 754-758.

(11) Duval, R.; Duplais, C. Fluorescent natural products as probes and tracers in biology. Nat. Prod. Rep. 2017, 34, 161-193.

(12) Tasior, M.; Kim, D.; Singha, S.; Krzeszewski, M.; Ahn, K. H.; Gryko, D. T. $\pi$-Expanded coumarins: synthesis, optical properties and applications. J. Mater. Chem. C 2015, 3, 1421-1446.

(13) Cao, D.; Liu, Z.; Verwilst, P.; Koo, S.; Jangjili, P.; Kim, J. S.; Lin, W. Coumarin-Based Small-Molecule Fluorescent Chemosensors. Chem. Rev. 2019, 119, 10403-10519.

(14) Ungureanu, I. M.; Van Ommeren, E.; Givaudan, S. A. Switzerland Off-taste masking sweetener. PCT Int. Appl. WO 2010100158 A1 20100910, Sep. 10, 2010; Chem. Abstr. 2010, 153, 381750.

(15) Orlow, S. J.; Komatsu, L. N. (New York University, USA) Coumarins as melanogenesis modifiers for cosmetics and pharmaceuticals. PCT Int. Appl. WO 2012103487 A1 20120802, Jan. 27, 2017; Chem. Abstr. 2012, 157, 305798.

(16) Guan, A.-Y.; Liu, C.-L.; Li, M.; Zhang, H.; Li, Z.-N.; Li, Z.-M. Design, synthesis and structure-activity relationship of novel coumarin derivatives. Pest Manage. Sci. 2011, 67, 647-655.

(17) Stefanachi, A.; Leonetti, F.; Pisani, L.; Catto, M.; Carotti, A. Coumarin: A Natural, Privileged and Versatile Scaffold for Bioactive Compounds. Molecules 2018, 23, 250-284.

(18) Yu, M.; Liu, X.; De Clercq, E. NF- $\kappa$ B: the inducible factors of HIV-1 transcription and their inhibitors. Mini-Rev. Med. Chem. 2009, 9, 60-69.

(19) Verotta, L.; Lovaglio, E.; Vidari, G.; Finzi, P. V.; Neri, M. G.; Raimondi, A.; Parapini, S.; Taramelli, D.; Riva, A.; Bombardelli, E. 4Alkyl- and 4-phenylcoumarins from Mesua ferrea as promising multidrug resistant antibacterials. Phytochemistry 2004, 65, 28672879.

(20) Pierson, J.-T.; Dumètre, A.; Hutter, S.; Delmas, F.; Laget, M.; Finet, J.-P.; Azas, N.; Combes, S. Synthesis and antiprotozoal activity of 4-arylcoumarins. Eur. J. Med. Chem. 2010, 45, 864-869.

(21) Combes, S.; Barbier, P.; Douillard, S.; McLeer-Florin, A.; Bourgarel-Rey, V.; Pierson, J.-T.; Fedorov, A. Y.; Finet, J. P.; Boutonnat, J.; Peyrot, V. Synthesis and Biological Evaluation of 4Arylcoumarin Analogues of Combretastatins. Part 2. J. Med. Chem. 2011, 54, 3153-3162.
(22) Revankar, H. M.; Bukhari, S. N. A.; Kumar, G. B.; Qin, H.-L. Coumarins scaffolds as COX inhibitors. Bioorg. Chem. 2017, 71, 146159.

(23) Medina, F. G.; Marrero, J. G.; Macías-Alonso, M.; González, M. C.; Córdova-Guerrero, I.; Teissier García, A. G.; Osegueda-Robles, S. Coumarin heterocyclic derivatives: chemical synthesis and biological activity. Nat. Prod. Rep. 2015, 32, 1472-1507.

(24) Rodrigues, J. L.; Rodrigues, L. R. Biosynthesis and heterologous production of furanocoumarins: perspectives and current challenges. Nat. Prod. Rep. 2021, 38, 869-879.

(25) Garazd, M. M.; Garazd, Y. L.; Khilya, V. P. Neoflavones. 2. Methods for synthesizing and modifying 4-arylcoumarins. Chem. Nat. Compd. 2005, 41, 245-271.

(26) Fedorov, A. Y.; Nyuchev, A. V.; Beletskaya, I. P. Catalytic methods of creation and functionalization of the coumarin skeleton. Chem. Heterocycl. Comp. 2012, 48, 166-178.

(27) Vekariya, R. H.; Patel, H. D. Recent Advances in the Synthesis of Coumarin Derivatives via Knoevenagel Condensation: A Review. Synth. Commun. 2014, 44, 2756-2788.

(28) Li, J.; Chen, H.; Zhang-Negrerie, D.; Du, Y.; Zhao, K. Synthesis of coumarins via $\mathrm{PIDA} / \mathrm{I}_{2}$-mediated oxidative cyclization of substituted phenylacrylic acids. RSC Adv. 2013, 3, 4311-4320.

(29) Singh, J.; Sharma, A. Visible Light-Induced Synthesis of Functionalized Coumarins. Adv. Synth. Catal. 2021, 363, 3411-3438.

(30) de Meijere, A.; Bräse, S.; Oestreich, M. Eds. Metal-Catalyzed Cross-Coupling Reactions and More 3 Volume Set; Wiley-VCH: Wenheim, Germany, 2014.

(31) Larhed, M. Ed. Science of Synthesis: Cross Coupling and HeckType Reactions 3, Metal-Catalyzed Heck-Type Reactions and C-C CrossCoupling via C-H Activation; Thieme: Stuttgart, Germany, 2013.

(32) Priyanka, S. R. K.; Katiyar, D. Recent Advances in TransitionMetal-Catalyzed Synthesis of Coumarins. Synthesis 2016, 48, 23032322.

(33) Catellani, M.; Chiusoli, G. P.; Marzolini, G.; Rossi, E. Designing a catalytic synthesis of 4-methylcoumarin from orthoiodophenyl 3-butenoate: ring closure and isomerization control. J. Organomet. Chem. 1996, 525, 65-69.

(34) Battistuzzi, G.; Cacchi, S.; De Salve, I.; Fabrizi, G.; Parisi, L. M. Synthesis of Coumarins in a Molten $n-\mathrm{Bu}_{4} \mathrm{NOAc} / n-\mathrm{Bu}_{4} \mathrm{NBr}$ Mixture through a Domino Heck Reaction/Cyclization Process. Adv. Synth. Catal. 2005, 347, 308-312.

(35) Ulgheri, F.; Marchetti, M.; Piccolo, O. Enantioselective Synthesis of $(S)$ - and $(R)$-Tolterodine by Asymmetric Hydrogenation of a Coumarin Derivative Obtained by a Heck Reaction. J. Org. Chem. 2007, 72, 6056-6059.

(36) Chen, J.; Liu, W.; Zhou, L.; Zhao, Y. Palladium catalyzed Heckarylation/cyclization cascade: An environmentally benign and efficient synthesis of 4-arylcoumarins in water. Tetrahedron Lett. 2018, 59, $2526-2531$.

(37) Queiroz, M.-J. R. P.; Abreu, A. S.; Calhelha, R. C.; Carvalho, M. S. D.; Ferreira, P. M. T. New strategies for the synthesis of heteroannulated 2-pyridinones, substituted 2-quinolinones and coumarins from dehydroamino acid derivatives. Tetrahedron 2008, $64,5139-5146$.

(38) Kadnikov, D. V.; Larock, R. C. Palladium-Catalyzed Carbonylative Annulation of Internal Alkynes: Synthesis of 3,4-Disubstituted Coumarins. J. Org. Chem. 2003, 68, 9423-9432.

(39) Kadnikov, D. V.; Larock, R. C. Palladium-catalyzed carbonylative annulation of terminal alkynes: synthesis of coumarins and 2quinolones. J. Organomet. Chem. 2003, 687, 425-435.

(40) Wu, X.-F.; Wu, L.; Jackstell, R.; Neumann, H.; Beller, M. A general palladium-catalyzed carbonylative synthesis of chromenones from salicylic aldehydes and benzyl chlorides. Chem. - Eur. J. 2013, 19, 12245-12248.

(41) Wienhold, M.; Molloy, J. J.; Daniliuc, C. G.; Gilmour, R. Coumarins by Direct Annulation: $\beta$-Borylacrylates as Ambiphilic C3Synthons. Angew. Chem., Int. Ed. 2021, 60, 685-689. 
(42) Lei, A.; Shi, W.; Liu, W.; Zhang, H.; He, C. Oxidative CrossCoupling Reactions; Wiley-VCH: Weinheim, 2016, DOI: 10.1002/ 9783527680986.

(43) Ferguson, J.; Zeng, F.; Alper, H. Synthesis of Coumarins via PdCatalyzed Oxidative Cyclocarbonylation of 2-Vinylphenols. Org. Lett. 2012, 14, 5602-5605.

(44) Sasano, K.; Takaya, J.; Iwasawa, N. Palladium(II)-Catalyzed Direct Carboxylation of Alkenyl C-H Bonds with $\mathrm{CO}_{2}$. J. Am. Chem. Soc. 2013, 135, 10954-10957.

(45) Yamamoto, Y. Synthesis of Heterocycles via Transition-MetalCatalyzed Hydroarylation of Alkynes. Chem. Soc. Rev. 2014, 43, $1575-1600$

(46) Trost, B. M.; Toste, F. D.; Greenman, K. Atom Economy Palladium-Catalyzed Formation of Coumarins by Addition of Phenols and Alkynoates via a Net C-H Insertion. J. Am. Chem. Soc. 2003, 125, $4518-4526$.

(47) Aoki, S.; Oyamada, J.; Kitamura, T. Formation of Coumarins by Palladium(II)-Catalyzed Reaction of Phenols with Ethyl Acrylates. Bull. Chem. Soc. Jpn. 2005, 78, 468-472.

(48) Sharma, U.; Naveen, T.; Maji, A.; Manna, S.; Maiti, D. Palladium-Catalyzed Synthesis of Benzofurans and Coumarins from Phenols and Olefins. Angew. Chem., Int. Ed. 2013, 52, 12669-12673.

(49) Zhang, X.-S.; Li, Z.-W.; Shi, Z.-J. Palladium-Catalyzed BaseAccelerated Direct C-H Bond Alkenylation of Phenols to Synthesize Coumarin Derivatives. Org. Chem. Front. 2014, 1, 44-49.

(50) Kim, D.; Min, M.; Hong, S. One-pot catalysis of dehydrogenation of cyclohexanones to phenols and oxidative Heck coupling: expedient synthesis of coumarins. Chem. Commun. 2013, 49, 40214023.

(51) Suna, E.; Shubin, K. Intramolecular Coupling via $\mathrm{C}\left(\mathrm{sp}^{2}\right)-\mathrm{H}$ Activation. In Science of Synthesis. Cross Coupling and Heck-Type Reactions 3. Metal-Catalyzed Heck-Type Reactions and C-H Couplings via C-H Activation; Larhed, M., Ed.; Georg Thieme Verlag: Stuttgart, 2013; pp. 643-653.

(52) Carral-Menoyo, A.; Sotomayor, N.; Lete, E. Palladiumcatalysed Heck-type alkenylation reactions in the synthesis of quinolines. Mechanistic insights and recent applications. Catal. Sci. Technol. 2020, 10, 5345-5361.

(53) Ortiz-de-Elguea, V.; Sotomayor, N.; Lete, E. Two consecutive Palladium(II)-promoted C-H alkenylation reactions for the synthesis of 3-alkenylquinolones. Adv. Synth. Catal. 2015, 357, 463-473.

(54) Carral-Menoyo, A.; Sotorríos, L.; Ortiz-de-Elguea, V.; DiazAndrés, A.; Sotomayor, N.; Gómez-Bengoa, E.; Lete, E. Intramolecular Palladium(II)-Catalyzed 6-endo $\mathrm{C}-\mathrm{H}$ Alkenylation Directed by the Remote N-Protecting Group: Mechanistic Insight and Application to the Synthesis of Dihydroquinolines. J. Org. Chem. 2020, 85, 2486-2503.

(55) Sandhu, S.; Bansal, Y.; Silakari, O.; Bansal, G. Coumarin hybrids as novel therapeutic agents. Bioorg. Med. Chem. 2014, 22, $3806-3814$

(56) Carral-Menoyo, A.; Misol, A.; Gómez-Redondo, M.; Sotomayor, N.; Lete, E. Palladium(II)-Catalyzed Intramolecular CH Alkenylation for the Synthesis of Chromanes. J. Org. Chem. 2019, 84, 2048-2060.

(57) Carral-Menoyo, A.; Ortiz-de-Elguea, V.; Martinez-Nunes, M.; Sotomayor, N.; Lete, E. Palladium-Catalyzed Dehydrogenative Coupling: An Efficient Synthetic Strategy for the Construction of the Quinoline Core. Mar. Drugs 2017, 15, 276.

(58) García-Rubia, A.; Urones, B.; Gómez-Arrayás, R.; Carretero, J. C. Pd ${ }^{\mathrm{II}}$-Catalyzed C-H Olefination of $\mathrm{N}$-(2-Pyridyl)sulfonyl Anilines and Arylalkylamines. Angew. Chem., Int. Ed. 2011, 50, 10927-10931.

(59) Urones, B.; Gomez-Arrayas, R.; Carretero, J. C. Pd ${ }^{\mathrm{II}}$-Catalyzed Di-o-olefination of Carbazoles Directed by the Protecting N-(2Pyridyl)sulfonyl Group. Org. Lett. 2013, 15, 1120-1123.

(60) García-Rubia, A.; Laga, E.; Cativiela, C.; Urriolabeitia, E. P.; Gómez-Arrayás, R.; Carretero, J. C. Pd-Catalyzed Directed ortho-C-H Alkenylation of Phenylalanine Derivatives. J. Org. Chem. 2015, 80, $3321-3331$
(61) Schiffner, J. A.; Oestreich, M. All-Carbon-Substituted Quaternary Carbon Atoms in Oxindoles by an Aerobic Palladium(II)-Catalyzed Ring Closure onto Tri- and Tetrasubstituted Double Bonds. Eur. J. Org. Chem. 2011, 1148-1154.

(62) Gordo, J.; Avó, J.; Parola, A. J.; Lima, J. C.; Pereira, A.; Branco, P. S. Convenient Synthesis of 3-Vinyl and 3-Styryl Coumarins. Org. Lett. 2011, 13, 5112-5115.

(63) Avó, J.; Martins, S.; Parola, A. J.; Lima, J.; Ramalho, J.; Branco, P.; Pereira, A. A Family of Styryl Coumarins: Synthesis, Spectroscopic, Photophysical and Photechemical Properties. ChemPlusChem 2013, 78, 789-792.

(64) Wang, X.; Li, S.; Pan, Y.; Wang, H.; Chen, Z.; Huang, K. Regioselective Palladium-Catalyzed Decarboxylative Cross-Coupling Reaction of Alkenyl Acids with Coumarins: Synthesis of 3Styrylcoumarin Compounds. J. Org. Chem. 2015, 80, 2407-2412.

(65) Jafarpour, F.; Zarei, S.; Barzegar Amiri Olia, M.; Jalalimanesh, N.; Rahiminejadan, S. Palladium-Catalyzed Decarboxylative CrossCoupling Reactions: A Route for Regioselective Functionalization of Coumarins. J. Org. Chem. 2013, 78, 2957-2964.

(66) Min, M.; Kima, Y.; Hong, S. Regioselective palladium-catalyzed olefination of coumarins via aerobic oxidative Heck reactions. Chem. Commun. 2013, 49, 196-198.

(67) Lavis, L. D.; Raines, R. T. Bright Ideas for Chemical Biology. ACS Chem. Biol. 2008, 3, 142-155.

(68) Lavis, L. D.; Raines, R. T. Bright Building Blocks for Chemical Biology. ACS Chem. Biol. 2014, 9, 855-866.

(69) Gao, Z.; Hao, Y.; Zheng, M.; Chen, Y. A fluorescent dye with large Stokes shift and high stability: synthesis and application to live cell imaging. RSC Adv. 2017, 7, 7604-7609.

(70) Boland, G. M.; Donnelly, D. M. X.; Finet, J.-P.; Rea, M. D. Synthesis of neoflavones by Suzuki arylation of 4-substituted coumarins. J. Chem. Soc. Perkin. Trans. 1 1996, 21, 2591-2597.

(71) Prateeptongkum, S.; Duangdee, N.; Thongyoo, P. Facile Iron(III) Chloride Hexahydrate Catalyzed Synthesis of Coumarins. ARKIVOC 2015, 2015, 248-258.

(72) Sekino, E.; Kumamoto, T.; Tanaka, T.; Ikeda, T.; Ishikawa, T. Concise Synthesis of Anti-HIV-1 Active (+)-Inophyllum B and (+)-Calanolide A by Application of (-)-Quinine-Catalyzed Intramolecular Oxo-Michael Addition. J. Org. Chem. 2004, 69, 2760-2767.

(73) Crecente-Campo, J.; Pilar Vázquez-Tato, M.; Seijas, J. A. Microwave-Promoted, One-Pot, Solvent-Free Synthesis of 4-Arylcoumarins from 2-Hydroxybenzophenones. Eur. J. Org. Chem. 2010, 4130-4135.

(74) Tang, B.-C.; Wang, M.; Ma, J.-T.; Wang, Z.-X.; Wu, Y.-D.; Wu, A.-X. Palladium-Acid Co-Catalyzed Cleavage of Alkynoates to Construct Dibenzo $[c, h]$ xanthene Derivatives. Adv. Synth. Catal. 2018, 360, 4023-4028.

(75) Zhao, B.; Xu, B. Visible-Light Promoted Oxidative Cyclization of Cinnamic Acid Derivatives using Xanthone as the Photocatalyst. Org. Biomol. Chem. 2021, 19, 568-573. 